\title{
5-sparse Steiner Triple Systems of Order $n$ Exist for Almost All Admissible $n$
}

\author{
Adam Wolfe* \\ Department of Mathematics \\ The Ohio State University, Columbus, OH, USA \\ water@math.ohio-state.edu
}

Submitted: Aug 5, 2003; Accepted: Nov 7, 2005; Published: Dec 5, 2005

Mathematics Subject Classification: 05B07

\begin{abstract}
Steiner triple systems are known to exist for orders $n \equiv 1,3 \bmod 6$, the admissible orders. There are many known constructions for infinite classes of Steiner triple systems. However, Steiner triple systems that lack prescribed configurations are harder to find. This paper gives a proof that the spectrum of orders of 5-sparse Steiner triple systems has arithmetic density 1 as compared to the admissible orders.
\end{abstract}

\section{Background}

Let $v \in \mathbb{N}$ and let $V$ be a $v$-set. A Steiner triple system of order $v$, abbreviated $\operatorname{STS}(v)$, is a collection $B$ of 3 -sets of $V$, called blocks or triples, such that every distinct pair of elements of $V$ lies in exactly one triple of $B$. An $\operatorname{STS}(v)$ exists exactly when $v \equiv 1$ or $3 \bmod 6$, the admissible orders. Wilson [13] showed that the number of non-isomorphic Steiner triple systems of order $n$ is asymptotically at least $\left(e^{-5} n\right)^{n^{2} / 6}$. Much less is known about the existence of Steiner triple systems that avoid certain configurations. An $r$ configuration of a system is a set of $r$ distinct triples whose union consists of no more than $r+2$ points. A Steiner triple system that lacks $r$-configurations is said to be $r$-sparse. In other words, a Steiner triple system where the union of every $r$ distinct triples has at least $r+3$ points is $r$-sparse.

In 1976, Paul Erdős conjectured that for any $r>1$, there exists a constant $N_{r}$ such that whenever $v>N_{r}$ and $v$ is an admissible order, an $r$-sparse $\operatorname{STS}(v)$ exists[4]. The statement is trivial for $r=2,3$. For $r=4$, there is only one type of 4-configuration, a Pasch. Paschs have the form:

$$
\{a, b, c\},\{a, d, e\},\{f, b, d\},\{f, c, e\}
$$

\footnotetext{
${ }^{*}$ Thanks to the editors of this journal for considering this for publication.
} 
In this paper, Paschs are written in the order presented above. Viewing a Steiner triple system as a 3-regular hypergraph with the point-set of the graph being the points of the Steiner triple system and the edge-set being the triples, we can graphically represent the system by plotting the point set as vertices and connecting the three vertices of an edge (triple) by a smooth line. With this in mind, a Pasch as in (1) can be graphically represented as:

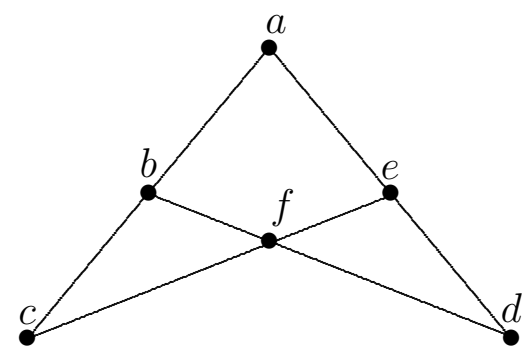

4-sparse, or anti-Pasch, Steiner triple systems were shown to exists for all admissible orders $v$ except for $v=7,13[6]$.

There are two types of 5 -configurations where the 5 blocks in the configuration contain 7 points, mias and mitres. A mia comes from a Pasch with the addition of an extra triple containing one new point not in the Pasch:

$$
\{a, b, c\},\{a, d, e\},\{f, b, d\},\{f, c, e\},\{a, f, g\} .
$$

A mitre has the form

$$
\{a, b, c\},\{a, d, e\},\{a, f, g\},\{b, d, f\},\{c, e, g\} .
$$

The element $a$ that occurs in three of the triples of the mitre is called the center of the mitre. A mitre as in (2) has the graphical representation as:

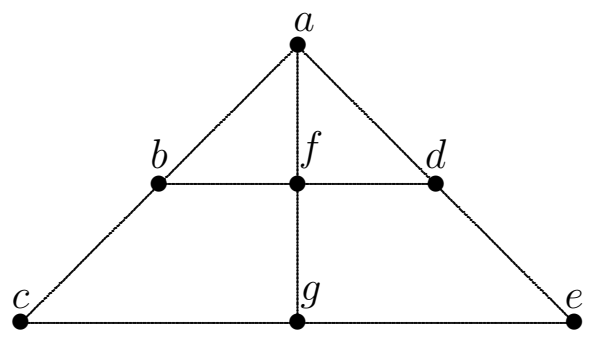

Generally, mitre configurations in this paper are written out with the first three triples being the triples with the center and the first three elements of the first three triples are the center. Steiner triple systems that do not contain mitres are called anti-mitre. Since all 5-configurations are derived from mitres or Paschs, 5-sparse Steiner triple systems are exactly those systems that are both 4-sparse (anti-Pasch) and anti-mitre.

Here is an outline of what the various sections of this article covers:

- Section 2 introduces meager systems and how they relate to 5-sparse Steiner triple systems. 
- Section 3 describes meager systems of order $m n+2$ for many values of $m$ and $n$.

- Section 4 introduces super-disjoint Steiner triple systems and provides an example of such systems of order $3 n$ under certain restrictions for $n$.

- Section 5 introduces average-free Steiner triple systems and manipulates the superdisjoint Steiner triple system from Section 4 to form an infinite class of average-free 5-sparse Steiner triple systems.

- By using an analytic technique on the results of the earlier sections, Section 6 shows that the spectrum of 5-sparse Steiner triple systems admit almost all admissible numbers.

Here is a list of known results on orders of 5-sparse Steiner triple systems:

Definition 1.1. Let $G$ be a finite abelian group. A Steiner triple system on $G$ is said to be transitive if whenever $\{x, y, z\}$ is a triple, then so is $\{x+a, y+a, z+a\}$ for $\{x, y, z, a\} \in G$. If the group is cyclic, then the Steiner triple system is referred to as cyclic Note that this definition can be extended to Latin squares (cf Definition 1.6) as well.

Theorem 1.2. (Colbourn, Mendelsohn, Rosa and S̆irán̆) [2] Transitive 5-sparse Steiner triple systems exists of order $v=p^{n}$ where $p$ is a prime, $p \equiv 19 \bmod 24$.

Theorem 1.3. (Ling) [10] If there exists a transitive 5-sparse $\operatorname{STS}(u), u \equiv 1 \bmod 6$ and a 5-sparse $\operatorname{STS}(v)$, then there exists a 5-sparse $\operatorname{STS}(u v)$.

Theorem 1.4. (Fujiwara) [5] There exists 5-sparse Steiner triple systems of order $n \equiv$ $1,19 \bmod 54$ except possibly for $n=109$.

We also have many small orders of 5-sparse Steiner triple systems realized:

Theorem 1.5. (Colbourn, Mendelsohn, Rosa and S̆irán̆) [2] Transitive 5-sparse STS(v) exist for admissible orders $v, 33 \leq v \leq 97$ and $v=19$.

Here are some definitions that we use in the following sections:

Definition 1.6. A Latin square of order $n$ is an $n \times n$ matrix $M=\left(m_{x y}\right)$ with entries from an $n$-set $V$, where every row and every column is a permutation of $V$. Labeling the rows and columns by $V$, it is convenient to view a Latin square as a pair $(V, B)$, where $B$ is a set of ordered triples on $V$ such that $(x, y, z) \in B$ if and only if $m_{x y}=z$ for $x, y, z \in V$.

Definition 1.7. A symmetric Latin square of order $n$ is a Latin square $(V, B)$ such that whenever $(x, y, z) \in B$ then so is the triple $(y, x, z) \in B$.

Definition 1.8. A partial Latin square of order $n$ is a triple system $(V, B)$ obtained from a partial $n \times n$ matrix with entries from an $n$-set $V$, where every element of $V$ appears in each row at most once and in each column at most once. 
Definition 1.9. A triple $(x, y, z)$ of a Latin square or a partial Latin square $(V, B)$ is called super-symmetric if all the permutations of the triple $(x, y, z)$, i.e. $(x, y, z),(x, z, y)$, $(y, x, z),(y, z, x),(z, x, y)$ and $(z, y, x)$ are in $B$ as well.

Definition 1.10. An idempotent Latin square $(V, B)$ on a set $V$ is one with the property that $(x, x, x) \in B$ for all $x \in V$.

Definition 1.11. We define a deleted symmetric square on a set $V$ to be a partial Latin square that can be obtained from an idempotent, symmetric Latin square $(V, B)$ by removing the triples $(x, x, x)$ for all $x \in V$.

Definition 1.12. Two deleted symmetric squares $\left(V, B_{1}\right)$ and $\left(V, B_{2}\right)$ on an $n$-set $V$ are said to be really disjoint if $B_{1} \bigcap B_{2}=\emptyset$ and for all $(x, y, z) \in B_{1}$, none of the six permutations of $\{x, y, z\}$ is in $B_{2}$.

\section{Meager Systems}

Definition 2.1. A (partial) Latin square on a set $V$ that has no subsquares of order 2, i.e. does not contain four triples of the form:

$$
(x, y, z),(x, a, b),(w, y, b),(w, a, z)
$$

for $x, y, z, w, a, b \in V$ is said to be $N_{2}$.

Definition 2.2. Let $B_{0}, B_{1}$ and $B_{2}$ be $N_{2}$ deleted symmetric squares of order $n$, on an $n$-set $V$, where the index $i$ of the square $B_{i}$ is taken as an element of $\mathbb{Z} / 3 \mathbb{Z}$. If the systems avoid each of the following configurations:

$$
\begin{gathered}
(x, y, z) \in B_{0},(x, z, w) \in B_{1} \text { and }(x, w, y) \in B_{2} \\
(x, y, z),(x, z, y) \in B_{t},(y, z, x) \in B_{t+2} \\
(x, y, z),(y, v, x),(x, v, w) \in B_{t},(z, w, x) \in B_{t+1} \\
(x, y, z),(y, w, x) \in B_{t},,(x, z, v) \in B_{t+1} \text { and }(x, v, w) \in B_{t+2}
\end{gathered}
$$

where $x, y, z, v, w \in V$ and $t \in \mathbb{Z} / 3 \mathbb{Z}$, then the system is called a meager system of order $n$. We denote the system by $\left(V, B_{0}, B_{1}, B_{2}\right)$. If $B_{0}=B_{1}=B_{2}$, then we simply refer to $\left(V, B_{0}\right)$ as a meager square of order $n$.

Note that if $\left(V, B_{0}, B_{1}, B_{2}\right)$ is a meager system, then so is $\left(V, B_{t}, B_{t+1}, B_{t+2}\right)$ for any $t \in \mathbb{Z} / 3 \mathbb{Z}$.

The usefulness of meager systems in constructing 5-sparse Steiner triple systems is apparent by the following lemma:

Lemma 2.3. Suppose there is a meager system of order $n$. Then there exists a 5-sparse Steiner triple system of order $3 n$. 
Proof. Let $\left(V, B_{0}, B_{1}, B_{2}\right)$ be a meager system of order $n$. We construct a Steiner triple system of order $3 n$ on $\mathbb{Z} / 3 \mathbb{Z} \times V$ as follows: Include triples $\left\{t_{x}, t_{y},(t+1)_{z}\right\}$ for $(x, y, z) \in B_{t}$ and $t \in \mathbb{Z} / 3 \mathbb{Z}$ and triples $\left\{0_{x}, 1_{x}, 2_{x}\right\}$ for $x \in V$.

If there is a Pasch in the construction, then the Pasch must have one of the two forms:

1. $\{t, t, t+1\},\{t, t, t+1\},\{t, t, t+1\},\{t, t, t+1\}$ for some $t \in \mathbb{Z} / 3 \mathbb{Z}$

2. $\{0,1,2\},\{0,0,1\},\{2,1,1\},\{2,0,2\}$.

In the first case, the Pasch would have come from a subsquare of order 2 from $B_{t}$ which is impossible since $B_{t}$ is assumed to be $N_{2}$. In the second case, filling in the subscripts would lead to the Pasch

$$
\left\{0_{x}, 1_{x}, 2_{x}\right\},\left\{0_{x}, 0_{y}, 1_{z}\right\},\left\{2_{w}, 1_{x}, 1_{z}\right\},\left\{2_{w}, 0_{y}, 2_{x}\right\}
$$

but the last three triples give a configuration $Q$ which cannot happen. Thus there are no Paschs.

If there is a mitre in the construction, then without loss of generality, the mitre could only have one of the following forms:

1. $\{0,0,1\},\{0,1,0\},\{0,2,2\},\{0,1,2\},\{1,0,2\}$.

2. $\{0,1,2\},\{0,0,1\},\{0,0,1\},\{1,0,0\},\{2,1,1\}$.

3. $\{0,1,2\},\{0,1,0\},\{0,2,2\},\{1,1,2\},\{2,0,2\}$.

1. Form 1 holds. Filling in the subscripts in the first form gives us the mitre:

$$
\left\{0_{x}, 0_{y}, 1_{z}\right\},\left\{0_{x}, 1_{y}, 0_{z}\right\},\left\{0_{x}, 2_{y}, 2_{z}\right\},\left\{0_{y}, 1_{y}, 2_{y}\right\},\left\{1_{z}, 0_{z}, 2_{z}\right\} .
$$

Then we have $(x, y, z),(x, z, y) \in B_{0}$ and $(y, z, x) \in B_{2}$, but this is an $M_{1}$ configuration.

2. Form 2 holds. Filling in the subscripts in the second form gives us the mitre:

$$
\left\{0_{x}, 1_{x}, 2_{x}\right\},\left\{0_{x}, 0_{y}, 1_{z}\right\},\left\{0_{x}, 0_{v}, 1_{w}\right\},\left\{1_{x}, 0_{y}, 0_{v}\right\},\left\{2_{x}, 1_{z}, 1_{w}\right\} .
$$

Thus we have $(x, y, z),(y, v, x),(x, v, w) \in B_{0}$ and $(z, w, x) \in B_{1}$, but this is an $M_{2}$ configuration.

3. Form 3 holds. Lastly, filling in the subscripts for the third form gives us the mitre:

$$
\left\{0_{x}, 1_{x}, 2_{x}\right\},\left\{0_{x}, 1_{v}, 0_{z}\right\},\left\{0_{x}, 2_{w}, 2_{y}\right\},\left\{1_{a}, 1_{e}, 2_{d}\right\},\left\{2_{a}, 0_{c}, 2_{b}\right\} .
$$

Thus $(x, y, z),(y, w, x) \in B_{2},(x, z, v) \in B_{0}$ and $(x, v, w) \in B_{1}$ which is an $M_{3}$ configuration.

Hence the resulting Steiner triple system could not have any mitres as well. Thus it is 5-sparse. 
The meager system avoiding $M_{1}, M_{2}$ and $M_{3}$ configurations assured that no mitres will occur in the construction. The squares being $N_{2}$ and avoiding $Q$ configurations assured that the result will lack Paschs. ${ }^{1}$

It is easy to check that meager systems of order $m$ do not exist for any odd $m \leq 7$, however we will see in the following section that a plethora of meager systems exist.

\section{$3 m n+2$ Meager Construction}

In this section we give a construction of a meager system of order $m n+2$ from a 4 -sparse Steiner triple system of order $m+2$ where $n$ is any odd number, $n \geq 5$. We will utilize special Latin squares called Valek squares in the meager system constructions:

Definition 3.1. Let $V$ be an $n$-set and let $\infty$ be a point not in $V$. A Valek square of order $n$ on $V$ is a symmetric Latin square on $V$ that contains a transversal along its main diagonal, say $(x, x, \sigma(x))$ where $\sigma$ is some permutation on $V$, such that if the main diagonal entries were deleted and triples $\{(\infty, x, \sigma(x)) \mid x \in V\}$ were introduced to the Latin square, then the resulting partial Latin square of order $n+1$ will be $N_{2}$.

It turns out that Valek squares of order $n$ exist for all odd $n$ except for $n=3$. To see this, we use the fact that an idempotent symmetric $N_{2}$ Latin square of odd order $n$ is Valek if whenever $(x, y, z)$ and $(x, z, y)$ are triples in the square, then $x=y=z$.

Lemma 3.2. Valek squares of odd order $n$ exist whenever $3 \nmid n$.

Proof. Let $n$ be an odd number such that $3 \nmid n$. Consider the symmetric $N_{2}$ Latin square on $\mathbb{Z} / n \mathbb{Z}$ with triples $(x, y, z)$ where $2 z=x+y, x, y, z \in \mathbb{Z} / n \mathbb{Z}$. Note that if $(x, y, z)$ and $(x, z, y)$ are triples, then $3 x=3 y$ which implies that $x=y=z$.

To cover the remaining cases, we utilize the following lemma:

Lemma 3.3. If an idempotent Valek square of order $n$ exists, then an idempotent Valek square of order $3 n$ exists.

Proof. Let $(\mathbb{Z} / n \mathbb{Z}, T)$ be an idempotent Valek square of order $n$. Consider the following Latin square of order $3 n$ on $\mathbb{Z} / 3 \mathbb{Z} \times \mathbb{Z} / n \mathbb{Z}$. Include the triples:

1. $((i, x),(i, y),(i, z))$ for $(x, y, z) \in T, i \in \mathbb{Z} / 3 \mathbb{Z}$.

2. $((0, x),(1, y),(2, x+y))$

3. $((1, x),(0, y),(2, x+y))$

4. $((0, x),(2, y),(1, y-x+1))$

\footnotetext{
${ }^{1}$ The idea for using the forms $\{0,0,1\},\{1,1,2\}$ and $\{2,2,0\}$ to produce a 5 -sparse Steiner triple system came from a popular Bose construction for 4-sparse Steiner triple systems that can be found in [7] and generalized in [8].
} 
5. $((2, x),(0, y),(1, x-y+1))$

6. $((1, x),(2, y),(0, y-x+2))$

7. $((2, x),(1, y),(0, x-y+2))$

where $x, y, z \in \mathbb{Z} / n \mathbb{Z}$. It may be easier to visualize the Latin square with the following: Let $A, B$ and $C$ be the Latin squares on $\mathbb{Z} / n \mathbb{Z}$ with triples $(x, y, x+y),(x, y, y-x+1)$ and $(x, y, y-x+2)$, respectively for $x, y, z \in \mathbb{Z} / n \mathbb{Z}$. Let $B^{T}$ and $C^{T}$ be the transposes of the squares (i.e. the first two coordinates of the triples swapped). The constructed Latin square has the form:

\begin{tabular}{|c|c|c|}
\hline$(0, T)$ & $(2, A)$ & $(1, B)$ \\
\hline$(2, A)$ & $(1, T)$ & $(0, C)$ \\
\hline$\left(1, B^{T}\right)$ & $\left(0, C^{T}\right)$ & $(2, T)$ \\
\hline
\end{tabular}

Note that the square is symmetric. Also, since the Latin square projected to the first coordinate

\begin{tabular}{|l|l|l|}
\hline 0 & 2 & 1 \\
\hline 2 & 1 & 0 \\
\hline 1 & 0 & 2 \\
\hline
\end{tabular}

is $N_{2}$ and the Latin squares $T, A, B$, and $C$ are $N_{2}$, it follows that the constructed Latin square is $N_{2}$ as well. Furthermore, since $T$ is idempotent, then so is our construction. It remains to show that if $((a, x),(b, y),(c, z))$ and $((a, x),(c, z),(b, y))$ are triples in the constructed Latin square, then $a=b=c$ and $x=y=z$. Since this property holds along the diagonal, we can reduce to the cases where $(a, b, c) \in\{(0,1,2),(1,0,2),(2,0,1)\}$. So, assuming $((a, x),(b, y),(c, z))$ and $((a, x),(c, z),(b, y))$ are triples in the square, if $(a, b, c)=$ $(0,1,2)$, then $z=x+y$ and $y=z-x+1$ which cannot happen. If $(a, b, c)=(1,0,2)$, then $z=x+y$ and $y=z-x+2$ which cannot happen. Lastly, if $(a, b, c)=(2,0,1)$, then $z=x-y+1$ and $y=x-z+2$ which cannot happen.

Theorem 3.4. Let $n>3$ be an odd number. Then a Valek square of order $n$ exists.

Proof. We can apply lemma 3.2 to get a Valek square of order $n$ if $3 \nmid n$. If $n=9$, we have an idempotent Valek square of order 9 given by Table 1. For the remaining cases, we can apply lemma 3.3 recursively.

The upcoming meager system construction is based on a generalization of a Steiner triple system construction introduced in [12] and developed in [11] and independently discovered by C. Demeng. The generalization is as follows:

Lemma 3.5. Let $m$ and $n$ be odd numbers with $n>1$ and $m \geq 5$. Suppose there exists a 4-sparse Steiner triple system $\left(V \bigcup\left\{\infty_{1}, \infty_{2}\right\}, \mathcal{T}\right)$ of order $n+2$ and suppose there exists an $N_{2}$ deleted symmetric square on $\left(P \bigcup\left\{\infty_{1}, \infty_{2}\right\}, \mathcal{S}\right)$ of order $m+2$. Then there exists an $N_{2}$ deleted symmetric square of order $m n+2$ on $(V \times P) \bigcup\left\{\infty_{1}, \infty_{2}\right\}$. 


\begin{tabular}{|l|l|l|l|l|l|l|l|l|}
\hline 0 & 8 & 7 & 6 & 5 & 3 & 4 & 1 & 2 \\
\hline 8 & 1 & 3 & 0 & 6 & 2 & 7 & 5 & 4 \\
\hline 7 & 3 & 2 & 5 & 1 & 4 & 0 & 8 & 6 \\
\hline 6 & 0 & 5 & 3 & 2 & 7 & 8 & 4 & 1 \\
\hline 5 & 6 & 1 & 2 & 4 & 8 & 3 & 0 & 7 \\
\hline 3 & 2 & 4 & 7 & 8 & 5 & 1 & 6 & 0 \\
\hline 4 & 7 & 0 & 8 & 3 & 1 & 6 & 2 & 5 \\
\hline 1 & 5 & 8 & 4 & 0 & 6 & 2 & 7 & 3 \\
\hline 2 & 4 & 6 & 1 & 7 & 0 & 5 & 3 & 8 \\
\hline
\end{tabular}

Figure 1: Idempotent Valek Square of Order 9

Proof. Given the Steiner triple system and the $N_{2}$ deleted symmetric square as described in the hypothesis, we construct an $N_{2}$ deleted symmetric square on $(V \times P) \bigcup\left\{\infty_{1}, \infty_{2}\right\}$. Let $T$ be the element of $V$ such that $\left\{\infty_{1}, \infty_{2}, T\right\} \in \mathcal{T}$. Define the graph $G$ on $V \backslash\{T\}$ as the graph connecting $X$ to $Y$ if and only if $\left\{X, Y, \infty_{i}\right\} \in \mathcal{T}$ for some $i$. Then it is clear that $G$ is the union of a collection of disjoint even cycles. By traversing each cycle, we can create a set of ordered pairs $\Omega$ where $(X, Y) \in \Omega$ implies that $X$ is adjacent to $Y$ in $G$ and for every $X \in V \backslash T$ there is exactly one $Y$ and exactly one $Z$ in $V \backslash T$ such that $(X, Y) \in \Omega$ and $(Z, X) \in \Omega$. For each $(X, Y) \in \Omega$, define $R_{\{X, Y\}}$ as a Valek square of order $m$ on $P$. For each $\{X, Y, Z\} \in \mathcal{T}$ where $X, Y, Z \notin\left\{\infty_{1}, \infty_{2}\right\}$ choose an ordered triple from the elements $\{X, Y, Z\}$ say, $(X, Y, Z)$, and choose an $N_{2}$ Latin square of order $m, L_{X Y Z}$ on $P .^{2}$

Now create the deleted symmetric Latin square by including the following triples: (For each unordered triple below, include all six ordered triples from the same elements.)

1. $\left(T_{x}, T_{y}, T_{z}\right)$ for $(x, y, z) \in \mathcal{S}$

2. $\left(T_{x}, T_{y}, \infty_{i}\right)$ for $\left(x, y, \infty_{i}\right) \in \mathcal{S}$

3. $\left(T_{x}, \infty_{i}, T_{y}\right)$ for $\left(x, \infty_{i}, y\right) \in \mathcal{S}$

4. $\left(\infty_{i}, T_{x}, T_{y}\right)$ for $\left(\infty_{i}, x, y\right) \in \mathcal{S}$

5. $\left\{X_{a}, X_{b}, Y_{c}\right\}$ for $(X, Y) \in \Omega$ and $(a, b, c) \in R_{\{X, Y\}}$.

6. $\left\{X_{a}, Y_{b}, \infty_{i}\right\}$ for $(X, Y) \in \Omega$ with $\left\{X, Y, \infty_{i}\right\} \in \mathcal{T}$ and $(a, a, b) \in R_{\{X Y\}}$

7. $\left\{X_{a}, Y_{b}, Z_{c}\right\}$ for $(a, b, c) \in L_{X Y Z}$.

Comment: The first four types of triples can be viewed as a copy of $\mathcal{S}$. It is clear that the above triples form a deleted symmetric square. See [11] for more detail on this.

Note that the constructed square is actually $N_{2}$. To see this, suppose on the contrary that there is a subsquare of order 2 composed of four triples, say $D$. There cannot exist

\footnotetext{
${ }^{2}$ By [3] we know that $N_{2}$ Latin squares exist for all orders $m$ with $m \neq 2,4$.
} 
two triples of $D$ of type 1 to 4 otherwise the remaining two would also have come from types 1 to 4 and the subsquare would have been derived from a subsquare of order 2 from $\mathcal{S}$ which cannot happen. Between any two triples of the subsquare of order 2 , there is a point in common. Thus we only have the following cases to consider:

1. D has a triple of type 1 . Then there must be a triple of type 7 in $D$. Without loss of generality, we may take the form of this type 7 triple to be $(T, X, Y)$ for some $X, Y \in V$. Then the forms of the triples would be $(T, T, T),(T, X, Y),(W, T, Z)$, and $(W, Y, T)$ where $Z \in V$. Then these latter two triples are also of type 7 . Hence $Y=Z$ which is impossible since these triples come from the Steiner triple system triples of $\mathcal{T}$.

2. D has a triple of type 2,3 or 4. Without loss of generality, we can assume that the triple is of type 4 . Then the other triples must be of type 6 or 7 and the forms of the triples are: $\left(\infty_{i}, T, T\right),\left(\infty_{i}, X, Y\right),(W, T, Y)$, and $(W, X, T)$ where $X, Y, W \in V$. Then $X=Y$ which cannot happen.

3. D has no triples of type 1 to 4. Since the triples of the subsquare are each supersymmetric, we can view the triples as unordered triples and investigate whether there are any Paschs that arise:

4. The Pasch has a triple of type 6. Then there must be another triple of type 6 . Thus the forms of the triples must look like: $\left\{\infty_{i}, X, Y\right\},\left\{\infty_{i}, A, B\right\},\{W, X, B\}$, and $\{W, A, Y\}$ with $X, Y, A, B, W \in V$ and $(X, Y) \in \Omega$. Since $\mathcal{T}$ is $N_{2}$, it must be that $A, B$ are not distinct from $X, Y$. Thus, it follows that $\{A, B\}=\{X, Y\}$. So, without loss of generality, take $A=X$ and $B=Y$. Then the last two triples are from triples of type 5 and so $W \in\{X, Y\}$. In either case, filling in the subscripts would give us a subsquare of order 2 which contradicts $R_{\{X Y\}}$ being Valek.

5. The Pasch has no triple of type 6 and has a triple of type 5. The forms of the triples must look like:

$$
\{X, X, Y\},\{X, A, B\},\{W, X, B\},\{W, A, Y\}
$$

for some $X, Y, A, B, W \in V$ with $(X, Y) \in \Omega$. If the forms of the latter three triples are derived from triples of type 7 , then $A=X$ which cannot happen. Thus, without loss of generality we can assume $\{X, A, B\}$ is from a triple of type 5 . If $\{X, A, B\}=\{X, Z, Z\}$ for some $Z \in V$, then $W=Z$ and thus $X=Y$ which is impossible. Hence $\{X, A, B\}=\{X, X, Y\}$. Thus each triple has the form $\{X, X, Y\}$ Filling in the subscripts would give us a subsquare of order 2 from $R_{\{X, Y\}}$ without using the main diagonal, which is impossible.

6. The Pasch only has triples of type 7. Projecting the triples to the forms would give either all distinct triples - thus forming a Pasch from $\mathcal{T}$ which is impossible - or the triples are all the same, say, $\{X, Y, Z\}$. In this case, filling in the subscripts based on, say, $L_{X Y Z}$, would give us a subsquare of order 2 from $L_{X Y Z}$ which is a contradiction. 
Thus the construction gives us an $N_{2}$ deleted symmetric square.

Applying Lemma 3.5, we can produce meager systems:

Lemma 3.6. Let $m, n$ be odd numbers with $m \equiv 1,5 \bmod 6, m \geq 7, m \neq 11$ and $n \geq 5$. Then there exists a meager system of order $m n+2$

Proof. The proof of Lemma 3.6 involves carefully constructing three deleted symmetric squares as prescribed by Lemma 3.5. For details on this construction, please refer to the appendix.

\section{Super-Disjoint Steiner Triple Systems}

Let $(V, B)$ be a Steiner triple system of order $n$. There is a natural deleted symmetric square $(V, \hat{B})$ that comes from the Steiner triple system by replacing every unordered triple of $B$ with the corresponding six ordered ones. Formally, define the triples of the square $\hat{B}$, the derived system from $B$ as:

$$
\hat{B}=\{(x, y, z) \mid\{x, y, z\} \in B .\}
$$

Suppose we have three Steiner triple systems on an $n$-set $V$ with triple sets $B_{0}, B_{1}$ and $B_{2}$. Let us investigate what conditions must hold on the triple sets to ensure that $\left(V, \hat{B}_{0}, \hat{B}_{1}, \hat{B}_{2}\right)$ is a meager system. Notice that $B_{i}$ has no Paschs if and only if $\hat{B}_{i}$ is $N_{2}$. Also, every triple in $\hat{B}_{i}$ is super-symmetric. Thus the three systems avoid $M_{1}, M_{2}$ and $M_{3}$ configurations if and only if the triples are pairwise disjoint in the deleted symmetric squares and thus in the triple sets of the Steiner triple systems. $Q$ configurations are avoided in the squares if there are no configurations $\{x, y, z\} \in B_{0},\{x, y, w\} \in B_{1}$ and $\{x, z, w\} \in B_{2}$ for any $x, y, z, w \in V$. This motivates the following definition:

Definition 4.1. Three Steiner triple systems $\left(V, B_{0}\right),\left(V, B_{1}\right)$ and $\left(V, B_{2}\right)$ are said to be super-disjoint if the following two conditions hold:

1. The systems are pairwise disjoint (i.e. $B_{0} \bigcap B_{1}=B_{0} \bigcap B_{2}=B_{1} \bigcap B_{2}=\emptyset$ ).

2. There are no configurations $\{x, y, z\} \in B_{0},\{x, y, w\} \in B_{1}$ and $\{x, z, w\} \in B_{2}$ for any $x, y, z, w \in V$.

We refer to the configuration in (2) as a $Q_{\text {sym }}$ configuration. Notice that the definition of super-disjointness is independent from the order that the Steiner triple systems are taken. Below is a lemma that states the relation between super-disjoint Steiner triple systems and meager systems of derived deleted symmetric squares:

Lemma 4.2. Suppose we have three 4-sparse super-disjoint Steiner triple systems on a set $V$ with triple sets $B_{0}, B_{1}$ and $B_{2}$. Then $\left(V, \hat{B}_{0}, \hat{B}_{1}, \hat{B}_{2}\right)$ is a meager system. 
There are many different constructions for 4-sparse Steiner triple systems that can be utilized to produce infinite classes of three 4-sparse super-disjoint Steiner triple systems. ${ }^{3}$ We now look at one of these constructions:

Lemma 4.3. There exist three 4-sparse super-disjoint Steiner triple systems of order $3 n$ provided $7 \nmid n, n$ odd, $n \geq 9$ (and so, under such conditions, a meager system of order $3 n$ exists).

Proof. Given the above restrictions on $n$, we will construct three Steiner triple systems of order $3 n$ : Let $G$ be an abelian group of order $n$. The Steiner triple systems will be on the set $\mathbb{Z} / 3 \mathbb{Z} \times G$. Let us label the triples of the three systems as $B_{1}, B_{2}$ and $B_{3}$. Choose elements of $G: a_{i}, b_{i}$ and $c_{i}$ for $i \in \mathbb{Z} / 3 \mathbb{Z}$ such that:

$$
\begin{gathered}
a_{0}+a_{1}+a_{2}=0 \\
b_{0}+b_{1}+b_{2}=0 \\
c_{i}=\left(-a_{i}-b_{i}\right) / 2 \\
c_{i} \neq a_{j}+b_{k} \text { and } a_{i} \neq b_{i}
\end{gathered}
$$

where $i, j, k \in \mathbb{Z} / 3 \mathbb{Z}, i, j, k$ distinct.

(E.g., taking $G=\mathbb{Z} / n \mathbb{Z}$, and $a_{0}=1, a_{1}=2, a_{2}=-3, b_{i}=-a_{i}$, and $c_{i}=0$ for $i \in\{0,1,2\}$ satisfies the above conditions.)

The triples in $B_{1}$ are:

$$
\begin{aligned}
& \left\{t_{x}, t_{y},(t+1)_{z}\right\} \text { where } z=(x+y) / 2+a_{t} \text { for } t \in \mathbb{Z} / 3 \mathbb{Z} \\
& \left\{0_{x}, 1_{x+a_{0}}, 2_{x+a_{0}+a_{1}}\right\} \text { where } x, y, z \text { are distinct elements of } G
\end{aligned}
$$

The triples in $B_{2}$ are:

$$
\begin{aligned}
& \left\{t_{x}, t_{y},(t+1)_{z}\right\} \text { where } z=(x+y) / 2+b_{t} \text { for } t \in \mathbb{Z} / 3 \mathbb{Z} \\
& \left\{0_{x}, 1_{x+b_{0}}, 2_{x+b_{0}+b_{1}}\right\} \text { where } x, y, z \text { are distinct elements of } G
\end{aligned}
$$

and the triples in $B_{3}$ are:

$$
\begin{aligned}
& \left\{(t+1)_{x},(t+1)_{y}, t_{z}\right\} \text { where } z=(x+y) / 2+c_{t} \text { for } t \in \mathbb{Z} / 3 \mathbb{Z} \\
& \left\{2_{x}, 1_{x+c_{1}}, 0_{x+c_{0}+c_{1}}\right\} \text { where } x, y, z \text { are distinct elements of } G
\end{aligned}
$$

Since $a_{0}+a_{1}+a_{2}=0, b_{0}+b_{1}+b_{2}=0$ and $c_{0}+c_{1}+c_{2}=0$, it is clear that the above systems are Steiner triple systems. To show that the systems are 4-sparse, without loss of generality, it is enough to show that the first system is 4-sparse since I will only be using the fact that $a_{0}+a_{1}+a_{2}=0$ : Assume, to the contrary, that there is a Pasch configuration in the first system. Since no two triples of a Pasch are disjoint, it is clear that it may contain at most one triple of the form $\{0,1,2\}$. With this in mind, projecting the Pasch to its form, the only possible Paschs are:

\footnotetext{
${ }^{3}[11]$ is particularly useful as a source of 4-sparse Steiner triple system constructions to be manipulated as super-disjoint.
} 
1. $\{t, t, t+1\},\{t, t, t+1\},\{t, t, t+1\},\{t, t, t+1\}$ where $t \in \mathbb{Z} / 3 \mathbb{Z}$.

2. $\{0,1,2\},\{0,0,1\},\{1,1,2\},\{2,2,0\}$

Filling in the subscripts in the first case yields:

$$
\begin{aligned}
& \left\{t_{x}, t_{y},(t+1)_{(x+y) / 2+a_{t}}\right\},\left\{t_{x}, t_{z},(t+1)_{(x+z) / 2+a_{t}}\right\},\left\{t_{w}, t_{y},(t+1)_{(w+y) / 2+a_{t}}\right\} \\
& \left\{t_{w}, t_{z},(t+1)_{(w+z) / 2+a_{t}}\right\}
\end{aligned}
$$

where $x, y, z, w \in G$ with $w \neq x$ and the following equations hold:

$$
(x+z) / 2+a_{t}=(w+y) / 2+a_{t}
$$

and

$$
(x+y) / 2+a_{t}=(w+z) / 2+a_{t} .
$$

Thus $x+z=w+y$ and $w+z=x+y$. This implies that $2 x=2 w$ and so $x=w$ (since $n$ is odd), a contradiction.

For the last case, filling in the subscripts gives us the following Pasch:

$$
\left\{0_{x}, 1_{x+a_{0}}, 2_{x+a_{0}+a_{1}}\right\},\left\{0_{x}, 0_{y}, 1_{(x+y) / 2+a_{0}}\right\},\left\{1_{x+a_{0}}, 1_{(x+y) / 2+a_{0}}, 2_{z}\right\},\left\{2_{z}, 2_{x+a_{0}+a_{1}}, 0_{y}\right\}
$$

where $x, y, z \in G$ and $x \neq y$. Also, the following equations hold:

$$
z=\frac{x+a_{0}+(x+y) / 2+a_{0}}{2}+a_{1} \quad \text { and } \quad y=\frac{z+x+a_{0}+a_{1}}{2}+a_{2} .
$$

Eliminating the variable $z$ and simplifying yields the condition $7 x=7 y$ which implies that $x=y$ since $7 \nmid n$, a contradiction.

Lastly, we must show that the three Steiner triple systems are super-disjoint. Just by considering each system's form, it is clear that the third system of triples, $B_{3}$, is disjoint from $B_{1}$ and $B_{2}$ except for possibly the triples of the form $\{0,1,2\}$. Assuming $B_{3}$ has a triple of this form in common with $B_{1}$, then for some $x, y \in G$, we have: $\left\{0_{x+c_{0}+c_{1}}, 1_{x+c_{1}}, 2_{x}\right\}=\left\{0_{y}, 1_{y+a_{0}}, 2_{y+a_{0}+a_{1}}\right\}$. Then $-c_{0}=a_{0}$ which implies that $\left(a_{0}+\right.$ $\left.b_{0}\right) / 2=a_{0}$ and so $a_{0}=b_{0}$, a contradiction. A similar argument holds for showing that $B_{3}$ is disjoint from $B_{2}$. Also, since $a_{i} \neq b_{i}$ for $i \in \mathbb{Z} / 3 \mathbb{Z}$, it is clear that $B_{1}$ is disjoint from $B_{2}$. (Note that the triples of the form $\{0,1,2\}$ between any two systems do not even have two points in common.)

To see that a $Q_{\text {sym }}$ configuration does not exist between the three systems, let us assume on the contrary. Then the three triples from $B_{1}, B_{2}$ and $B_{3}$, respectively that form the $Q_{\text {sym }}$ configuration can have at most one triple of the form $\{0,1,2\}$ since any two triples of a $Q_{\text {sym }}$ configuration have two points in common. Writing the $Q_{\text {sym }}$ configuration as in Definition 4.1 we have the following cases of the forms of triples of the $Q_{\text {sym }}$ configuration from $B_{1}, B_{2}$ and $B_{3}$, respectively: 
1. $\{t, t+1, t+2\},\{t, t+1, t\},\{t, t+2, t\}$

2. $\{t, t+1, t\},\{t, t+1, t+2\},\{t, t, t+2\}$

3. $\{t, t, t+1\},\{t, t, t+1\},\{t, t+1, t+1\}$

where $t \in \mathbb{Z} / 3 \mathbb{Z}$. By swapping $B_{1}$ with $B_{2}$ if necessary, we only need to consider the first and third case. Filling in the subscripts in the first case gives us $\left\{t_{x},(t+1)_{x+a_{t}},(t+\right.$ $\left.2)_{x+a_{t}+a_{t+1}}\right\},\left\{t_{x},(t+1)_{(x+w) / 2+b_{t}}, t_{w}\right\},\left\{t_{x},(t+2)_{(x+w) / 2+c_{t+2}}, t_{w}\right\}$ for some $x, w \in G$ where $x+a_{t}=(x+w) / 2+b_{t}$ and $x+a_{t}+a_{t+1}=(x+w) / 2+c_{t+2}$. This implies that $c_{t+2}=b_{t}+a_{t+1}$, a contradiction.

Filling in the subscripts for the third case, we have:

$$
\left\{t_{x}, t_{y},(t+1)_{(x+y) / 2+a_{t}}\right\},\left\{t_{x}, t_{y},(t+1)_{(x+y) / 2+b_{t}}\right\},\left\{t_{x},(t+1)_{(x+y) / 2+a_{t}},(t+1)_{(x+y) / 2+b_{t}}\right\}
$$

for distinct elements $x, y \in G$ where

$$
\frac{(x+y) / 2+a_{t}+(x+y) / 2+b_{t}}{2}+c_{t}=x .
$$

This implies that $x=y$, a contradiction.

Hence the construction gives us a set of three super-disjoint 4-sparse Steiner triple systems of order $3 n$.

\section{Average-free 5-sparse Steiner Triple Systems}

This section gives a construction of a 5-sparse Steiner triple system of order $m n+2$ from a 5-sparse average-free Steiner triple system of order $m+2$ and a 5-sparse Steiner triple system of order $n+2$. Similar to the construction in Lemma 3.5, this upcoming construction is based on a construction in [11].

Definition 5.1. Let $G$ be an abelian group of odd order. Let $\left\{\infty_{1}, \infty_{2}\right\}$ be two points not in $G$. A Steiner triple system $\left(G \cup\left\{\infty_{1}, \infty_{2}\right\}, B\right)$ is said to be average-free (with respect to $G$ ) if there are no triples $\{x, y, z\} \in B$ where $x, y, z \in G$ and $2 z=x+y$. We also say that a triple $\{x, y, z\}$ is average-free if $2 z \neq x+y, 2 x \neq y+z$ and $2 y \neq x+z$ and an average triple is a triple that is not average-free.

The following analysis of $P_{\infty_{1}, \infty_{2}}$ is necessary before presenting the 5-sparse construction.

Definition 5.2. Let $(V, B)$ be a Steiner triple system containing two points $\infty_{1}$ and $\infty_{2}$. Let $t$ be the point in the Steiner triple system such that $\left\{t, \infty_{1}, \infty_{2}\right\} \in B$. Let $\Omega$ be a set of ordered pairs of elements from $V \backslash\left\{t, \infty_{1}, \infty_{2}\right\}$ such that if $(X, Y) \in \Omega$, then $\left\{X, Y, \infty_{i}\right\} \in B$ for some $i \in\{0,1\}$ and for every $X \in V \backslash\left\{t, \infty_{1}, \infty_{2}\right\}$ there is a unique $Z_{1}$ and a unique $Z_{2}$ where $\left(X, Z_{1}\right),\left(Z_{2}, X\right) \in \Omega$. Define a $P_{\Omega}$ configuration as a set of four triples of $B$ that looks like:

$$
\{X, Y, Z\},\{X, A, B\},\left\{Y, A, \infty_{i}\right\},\left\{Z, B, \infty_{j}\right\} .
$$

where $(A, Y),(B, Z) \in \Omega$ and $i, j \in\{1,2\}$. 
Lemma 5.3. Let $(V, B)$ be a 4-sparse Steiner triple system containing two points $\infty_{1}$ and $\infty_{2}$. Let $\Omega$ be as in Definition 5.2. Given a $P_{\Omega}$ configuration,

$$
\{X, Y, Z\},\{X, A, B\},\left\{Y, A, \infty_{i}\right\},\left\{Z, B, \infty_{j}\right\}
$$

with $(A, Y),(B, Z) \in \Omega$, there is at most one other $P_{\Omega}$ configuration that contains the triple $\{X, Y, Z\}$.

Proof. Assume that we have a $P_{\Omega}$ as above with the triple $\{X, Y, Z\}$. Without loss of generality, we can take $\left\{Y, A, \infty_{1}\right\}$ and $\left\{Z, B, \infty_{2}\right\}$ to be triples of $B$. Let $R, S, T, U$ be points of $V$ such that $\left\{X, R, \infty_{1}\right\},\left\{X, S, \infty_{2}\right\},\left\{Y, T, \infty_{2}\right\}$ and $\left\{Z, U, \infty_{1}\right\}$ are triples of $B$. It follows that $(Z, U),(Y, T) \in \Omega$. Since $B$ has no Paschs, there are only three possibilities of other $P_{\infty_{1}, \infty_{2}}$ with $\{X, Y, Z\}$ :

$$
\begin{array}{r}
\{X, U, T\},\{X, Z, Y\},\left\{Z, U, \infty_{1}\right\},\left\{Y, T, \infty_{2}\right\} \\
\{Y, U, S\},\{Y, Z, X\},\left\{Z, U, \infty_{1}\right\},\left\{X, S, \infty_{2}\right\} \\
\{Z, R, T\},\{Z, X, Y\},\left\{X, R, \infty_{1}\right\},\left\{Y, T, \infty_{2}\right\}
\end{array}
$$

The first and second configurations cannot exist simultaneously because then there would be a Pasch. Similarly, the first and third configurations together would lead to a Pasch. Lastly, the second and third configurations cannot both simultaneously exist since then $(X, R),(X, S) \in \Omega$ which implies that $R=S$ which cannot happen. Thus there can only be one other $P_{\Omega}$ configuration with the triple $\{X, Y, Z\}$.

The upcoming construction will have forms of triples derived from a $P_{\Omega}$ configuration that form a mitre. The subscripts must be chosen in a way to avoid such mitres. For this purpose we introduce three special $N_{2}$ Latin squares:

Definition 5.4. Let $G$ be an abelian group of odd order $m$. Then $G$ is either the cyclic group of order $m$ on $\mathbb{Z} / m \mathbb{Z}$ or we can express $G=H \times \mathbb{Z} / k \mathbb{Z}$ for some abelian group $H$ and some $k \geq 3$. In the former case define the Latin squares $L_{G}^{i}$ for $i=0,1,2$ as follows:

$$
L_{G}^{i}=\{(x, y, z) \mid x+y+\sigma(z)=6 i\}
$$

for $x, y, z \in \mathbb{Z} / m \mathbb{Z}$ where $\sigma$ is a permutation on $\mathbb{Z} / m \mathbb{Z}$ swapping $0 \leftrightarrow 2$ and $4 \leftrightarrow 6$. In the latter case, choose a non-zero element $r \in H$ and define $L_{G}^{i}$ as:

$$
L_{G}^{i}=\{(x, a),(y, b),(z, c) \mid 2 z=x+y+r \text { and } a+b+c=i\}
$$

for $x, y, z \in H$ and $a, b, c \in \mathbb{Z} / k \mathbb{Z}$.

Note that $L_{G}^{i}$ is $N_{2}$. Now we are ready to give the construction.

Lemma 5.5. Let $\left(\mathbb{Z} / n \mathbb{Z} \cup\left\{\infty_{1}, \infty_{2}\right\}, T\right)$ be a 5-sparse Steiner triple system of order $n+2$ with $n \geq 17$. Let $m \geq 17$ and $\left(G \cup\left\{\infty_{1}, \infty_{2}\right\}, S\right)$ be an average-free 5-sparse Steiner triple system with $G$ being an abelian group of odd order $m$ (so there are no triples $\{x, y, z\}$ of $S$ where $x, y, z \in G$ and $2 z=x+y)$. Then there exists an average-free 5-sparse Steiner triple system of order $m n+2$ on $(\mathbb{Z} / n \mathbb{Z} \times G) \cup\left\{\infty_{1}, \infty_{2}\right\}$. 
Proof. Assume that we have such 5-sparse systems as described in the hypothesis. Let $t$ be the element of $\mathbb{Z} / n \mathbb{Z}$ such that $\left\{t, \infty_{1}, \infty_{2}\right\}$ is a triple of $T$. For convenience, rearrange the points of $T$ as necessary so that any $\{t, X, Y\} \in T$ with $X, Y \in \mathbb{Z} / n \mathbb{Z}$ is average-free. (For example, we can remap $t$ to 1 and take the triples of $t$ to look like:

$$
\begin{aligned}
\{1, X,-X\} & \text { for each } X \notin\{0, \pm 1, \pm 1 / 3\} \\
\{1,0,1 / 3\} & \\
\{1,-1,-1 / 3\} &
\end{aligned}
$$

which works since $3 \nmid n$.) Let $L_{G}^{i}$ be as in Definition 5.4. For the triple set $T$, define $\Omega$ as in Definition 5.2. Consider the graph $K$ on $T$ where $\{X, Y, Z\}$ and $\{X, A, B\}$ are adjacent if and only if $X, Y, Z, A, B \notin\left\{\infty_{1}, \infty_{2}\right\}$ and $\{X, Y, Z\}$ and $\{X, A, B\}$ are together in a $P_{\Omega}$ configuration. By Lemma 5.3, the degree of every vertex in $K$ is at most 2 . Thus the graph has a proper vertex 3 -coloring. So let $f: T \rightarrow\{0,1,2\}$ be such a coloring. Let $s \in G$ be the element such that $\left\{s, \infty_{1}, \infty_{2}\right\} \in S$.

Define a set $W$ of ordered 3-tuples on $\mathbb{Z} / n \mathbb{Z} \backslash\{t\}$ as follows: For each triple of $\{X, Y, Z\} \in T$ such that $t, \infty_{1}, \infty_{2} \notin\{X, Y, Z\}$ choose an ordering on the triple, say $(X, Y, Z)$ such that if $2 Z=X+Y$, then the ordering must be $(X, Y, Z)$. Otherwise, it does not matter how the order is chosen. Include such ordered triples in $W$.

We construct a Steiner triple system $\left((\mathbb{Z} / n \mathbb{Z} \times G) \cup\left\{\infty_{1}, \infty_{2}\right\}, B\right)$ based on a construction in [11] as follows. Include seven types of triples in $B$ :

1. $\left\{t_{a}, t_{b}, t_{c}\right\}$ for $\{a, b, c\} \in S$ with $a, b, c \notin\left\{\infty_{1}, \infty_{2}\right\}$

2. $\left\{t_{a}, t_{b}, \infty_{i}\right\}$ for $\left\{a, b, \infty_{i}\right\} \in S$

3. $\left\{t_{s}, \infty_{1}, \infty_{2}\right\}$

4. $\left\{X_{a}, X_{b}, Y_{c}\right\}$ where $(X, Y) \in \Omega, a, b, c \in G$ and $2 c=a+b$

5. $\left\{X_{a}, Y_{a}, \infty_{i}\right\}$ where $\left\{X, Y, \infty_{i}\right\} \in T$ and $a \in G$

6. $\left\{X_{a}, Y_{b}, Z_{c}\right\}$ where $(X, Y, Z) \in W, f(\{X, Y, Z\})=i$ and $(a, b, c) \in L_{G}^{i}$

7. $\left\{t_{a}, X_{b}, Y_{c}\right\}$ where $\{t, X, Y\} \in T$ and $a+b+c=0$.

It is clear that $B$ does form a Steiner triple system of order $m n+2$ on $\mathbb{Z} / n \mathbb{Z} \times G) \cup$ $\left\{\infty_{1}, \infty_{2}\right\}$. To see that $B$ has no Paschs, define $\tilde{B}$ as the super-symmetric deleted symmetric square that is derived from $B$. Note that the subscript of the triples of type 4 come from an (idempotent) Valek square since $3 \nmid m$. Also, the subscripts of triples of type 6 and 7 come from $N_{2}$ Latin squares. Thus the triples of $\tilde{B}$ are an instance of the construction in Lemma 3.5. Thus, $\tilde{B}$ has no subsquares of order 2. It follows immediately that $B$ has no Paschs.

To see that $B$ has no mitres, assume on the contrary, that there is a mitre in $B$. The mitre cannot have more than two triples from the set of type $\{1,2,3\}$ since otherwise the subscripts of the mitre would have been derived from a mitre in $S$. With this in mind, consider the following cases: 
1. The center of the mitre is $\infty_{i}$ for some $i$. If there is a triple of type in $\{1,2,3\}$ in the mitre, then consider the following subcases:

(a) There is a triple containing a point $\infty_{j}(j \neq i)$. Then there must be a triple of type 3 . We can rearrange the mitre so that the form of the mitre looks like:

$$
\left\{\infty_{i}, \infty_{j}, t\right\},\left\{\infty_{i}, X, Y\right\},\left\{\infty_{i}, V, Z\right\},\left\{\infty_{j}, X, V\right\},\{t, V, Z\}
$$

where $t \notin\{X, Y, V, Z\}$. This comes from a mitre in $T$ which cannot happen.

(b) If there is a triple containing points of form $t$ but no points with $\infty_{j}$ where $j \neq i$, then we can take the form of the mitre to look like:

$$
\left\{\infty_{i}, t, t\right\},\left\{\infty_{i}, X, Y\right\},\left\{\infty_{i}, Z, V\right\},\{t, X, Z\},\{t, Y, V\} .
$$

where $t \notin X, Y, Z, V$. Filling in the subscripts gives us the mitre

$$
\left\{\infty_{i}, t_{c}, t_{d}\right\},\left\{\infty_{i}, X_{a}, Y_{a}\right\},\left\{\infty_{i}, Z_{b}, V_{b}\right\},\left\{t_{c}, X_{a}, Z_{b}\right\},\left\{t_{d}, Y_{a}, V_{b}\right\}
$$

where $a, b, c, d \in G$. Since the last two triples of the mitre are of type 7 , it follows that $c=d$ which cannot happen because of the first triple.

(c) There are no points of the form $t$ or $\infty_{j}$, with $j \neq i$. Then the forms of the triples cannot all be distinct since this would lead to a mitre in $T$. This leads to two possibilities for the form of the mitre. One of them is

$$
\left\{\infty_{i}, X, Y\right\},\left\{\infty_{i}, Y, X\right\},\left\{\infty_{i}, Z, Z\right\},\{X, Y, Z\},\{Y, X, Z\} .
$$

It follows that $Z=t$ which cannot happen by virtue of this case. The other possibility of the form is:

$$
\left\{\infty_{i}, X, Y\right\},\left\{\infty_{i}, X, Y\right\},\left\{\infty_{i}, R, S\right\},\{X, X, R\},\{Y, Y, S\} .
$$

where $X, Y, R, S$ are distinct elements and $t \notin\{X, Y, R, S\}$. It follows that the last two triples of the mitre came from type 4 . So it must be that $T$ has triples

$$
\left\{\infty_{j}, X, R\right\},\left\{\infty_{j}, Y, S\right\},\left\{\infty_{i}, X, Y\right\},\left\{\infty_{i}, R, S\right\}
$$

where $i \neq j$ which form a Pasch in $T$, a contradiction.

2. The center of the mitre is of form $t$. We have three subcases to consider:

(a) There is a point $\infty_{i}$ in the mitre for some $i$. Then the mitre must have the form:

$$
\left\{t, \infty_{1}, \infty_{2}\right\},\{t, X, Y\},\{t, Z, V\},\left\{\infty_{1}, X, Z\right\},\left\{\infty_{2}, Y, V\right\}
$$

where $X, Y, Z, V, t$ are all distinct. Then the form of the mitre comes from a mitre in $T$ which cannot happen. 
(b) There is a triple of type 1 in the mitre. Then the form of the mitre must look like:

$$
\{t, t, t\},\{t, X, Y\},\{t, Y, X\},\{t, X, Y\},\{t, Y, X\} .
$$

Filling in the subscripts gives us the mitre:

$$
\left\{t_{a}, t_{b}, t_{c}\right\},\left\{t_{a}, X_{x}, Y_{y}\right\},\left\{t_{a}, Y_{v}, X_{w}\right\},\left\{t_{b}, X_{x}, Y_{v}\right\},\left\{t_{c}, Y_{y}, X_{w}\right\}
$$

for some $a, b, c, x, y, z, w \in G$. Since the last four triples of the mitre are of type 7 , the following equations must hold:

$$
\begin{array}{r}
x+y+a=0 \\
v+w+a=0 \\
x+v+b=0 \\
y+w+c=0 .
\end{array}
$$

Then $2 a=b+c$ which cannot happen since $S$ is average-free.

(c) There are no triples of type 1,2 or 3 in the mitre. Then the form of the mitre must look like:

$$
\{t, X, Y\},\{t, Z, V\},\{t, A, B\},\{X, Z, A\},\{Y, V, B\} .
$$

If $X, Y, Z, V, A, B$ are all distinct, then the form of the mitre would have come from a mitre in $T$ which cannot happen. Thus, without loss of generality, we can assume that $Z \in\{X, Y\}$. If $Z=Y$, then $V=X$. Then it must be $A=B=t$ which cannot happen by virtue of the hypothesis of this case. Thus, take $Z=X$. Then $V=Y$. Thus we have the following form of mitre:

$$
\{t, X, Y\},\{t, X, Y\},\{t, A, B\},\{X, X, A\},\{Y, Y, B\} .
$$

It follows that there are distinct triples in $T$ :

$$
\{t, X, Y\},\{t, A, B\},\left\{\infty_{i}, X, A\right\},\left\{\infty_{j}, Y, B\right\}
$$

If $i=j$, then the above triples form a Pasch which cannot happen. However, if $i \neq j$, then appending the four triples with the triple $\left\{t, \infty_{1}, \infty_{2}\right\} \in T$ gives a mitre in $T$ which cannot happen.

3. The form of the center of the mitre is not in $\left\{t, \infty_{1}, \infty_{2}\right\}$. Consider the following subcases:

(a) There is a triple of type 1 in the mitre. Then the mitre must have the form:

$$
\{X, t, Y\},\{X, t, Y\},\{X, t, Y\},\{t, t, t\},\{Y, Y, Y\}
$$

for some $X, Y \in \mathbb{Z} / n \mathbb{Z}$. Then $Y=t$ which cannot happen in this case. 
(b) There is a triple of type 2 in the mitre. Then the mitre has the form:

$$
\left\{X, \infty_{i}, Y\right\},\{X, t, Z\},\{X, t, Z\},\left\{\infty_{i}, t, t\right\},\{Y, Z, Z\}
$$

for some $Y, Z \in \mathbb{Z} / n \mathbb{Z}$. Since $Y$ cannot equal $X$, it is clear that $T$ has a triple $\left\{\infty_{j}, Y, Z\right\}$ where $i \neq j$. Then there is a Pasch in $T$ :

$$
\left\{\infty_{i}, Y, X\right\},\left\{\infty_{i}, t, \infty_{j}\right\},\left\{Z, Y, \infty_{j}\right\},\{Z, t, X\}
$$

which cannot happen.

(c) There is a triple of type 3 in the mitre. Then the mitre has the form:

$$
\left\{X, \infty_{1}, Y\right\},\left\{X, \infty_{2}, Z\right\},\{X, T, V\},\left\{\infty_{1}, \infty_{2}, t\right\},\{Y, Z, V\}
$$

where $X, Y, Z, V \in \mathbb{Z} / n \mathbb{Z}$. Note that $X, Y, Z, V, t$ are distinct, but then the form is from a mitre in $T$ which cannot happen.

(d) There are no triples of type 1,2 or 3 but there are points $\infty_{1}, \infty_{2}$ in the mitre. Then the mitre has the form:

$$
\left\{X, \infty_{1}, Y\right\},\left\{X, Z, \infty_{2}\right\},\{X, A, B\},\left\{\infty_{1}, Z, A\right\},\left\{Y, \infty_{2}, B\right\}
$$

where $X, Y, A, B \in \mathbb{Z} / n \mathbb{Z}$. Since there are no mitres in $T$, it cannot be the case that $X, Y, A, B$ are all distinct. The only possibility is that the triple form $\{X, A, B\}$ is from a triple of type 4 . It follows that $A=B$, but then there is a triple $\left\{X, A, \infty_{i}\right\} \in T$ for some $i$ which clearly cannot happen.

(e) There are no triples of type 1,2, or 3, but there is exactly one point from $\left\{\infty_{1}, \infty_{2}\right\}$ in the mitre. Then the mitre has the form:

$$
\left\{X, \infty_{i}, Y\right\},\{X, A, C\},\{X, B, D\},\left\{\infty_{i}, A, B\right\},\{Y, C, D\}
$$

where $X, Y, A, B, C, D \in \mathbb{Z} / n \mathbb{Z}$ with $X \neq t$. Note that $X, Y, A, B, C, D$ cannot all be distinct. If $\{A, B\} \cap\{X, Y\} \neq \emptyset$, then without loss of generality, we can take $A=X$. Then $B=Y$. Then $\{C, D\}=\{X, Y\}$ which would imply that both $(X, Y),(Y, X) \in \Omega$ which cannot happen.

Since $A \neq B$, it follows that $A, B, X, Y$ are distinct. Thus, by swapping $C$ with $D$ if necessary, we can assume the following four cases:

i. $C=D$. Then $A=B$ which cannot happen.

ii. $C=A$. Then there is a Pasch

$$
\left\{X, Y, \infty_{i}\right\},\{X, B, D\},\{A, Y, D\},\left\{A, B, \infty_{i}\right\}
$$

in $T$ which cannot happen.

iii. $C=B$. Then $A=D$. It follows that $T$ contains the triples $\{X, A, C\}$ and $\left\{\infty_{i}, A, C\right\}$ which cannot happen. 
iv. $C=X$. Then $D \in\{X, Y\}$. It follows that $B \in\{X, Y\}$ but this case was already covered in the beginning of subcase $(e)$.

(f) There are only triples of type 4, 6 or 7 in the mitre. Then the mitre has the form:

$$
\{X, A, B\},\{X, C, D\},\{X, E, F\},\{A, C, E\},\{B, D, F\}
$$

where $X, A, B, C, D, E, F \in \mathbb{Z} / n \mathbb{Z}$ and they all cannot be distinct since otherwise we would have a mitre in $T$. With this in mind, consider the following cases:

i. At least one of the first three triples of the mitre is of type 4. Then, without loss of generality, we can assume that $X=A$ or $A=B$. If $X=A$, then $D=E$ and so $C=F$. Then $X=B$ which would imply that $X=t$ and that case was covered in case 2 . Consider $A=B$. It follows that $X \neq A$. If $C, D, E, F, X, A$ are all distinct, then there is a Pasch in $T$ :

$$
\{X, C, D\},\{X, F, E\},\{A, C, E\},\{A, F, D\}
$$

which cannot happen. So, without loss of generality, we can assume $C \in$ $\{X, A, D, E, F\}$. It is easy to see that each case works out to having $X=t$ and that case was covered in case 2 .

ii. There are no triples of type 4 in the first three triples. Thus the first three triples of the mitre are of type 6 or 7 . Thus the forms of elements in each of the first three triples are distinct. Since there are no mitres in $T$, the elements $X, A, B, C, D, E, F$ cannot all be distinct. Without loss of generality, we may assume that $A=D$ or $A=C$. In the former case we have $C=B$ and thus $X=E=F$ which would imply that $X=t$ and that case was covered in case 2 . Now consider $A=C$ and so $B=D$. So the last two triples of the mitre are of type 4 . It follows that the first three triples are of type 6. Filling in the subscripts gives us:

$$
\left\{X_{x}, A_{a}, B_{b}\right\},\left\{X_{x}, A_{c}, B_{d}\right\},\left\{X_{x}, E_{e}, F_{f}\right\},\left\{A_{a}, A_{c}, E_{e}\right\},\left\{B_{b}, B_{d}, F_{f}\right\}
$$

where $x, a, b, c, d, e, f \in G$. Since the forms of these triples are from a $P_{\Omega}$ configuration, it follows that the subscripts $\{x, a, b\}$ and $\{x, c, d\}$ come from two triples of $L_{G}^{i}$ and the subscripts $\{x, e, f\}$ come from a triple of $L_{G}^{j}$ for some $i \neq j$. To do further analysis, we must consider the following two cases: If $G$ is not a cyclic group of order $n$, then $G$ is viewed as $H \times \mathbb{Z} / k \mathbb{Z}$ as in Definition 5.4. For an element $a=(x, y) \in G$, with $x \in H$ and $y \in \mathbb{Z} / k \mathbb{Z}$, define $\hat{a}=y$. It follows that:

$$
\begin{aligned}
\hat{x}+\hat{a}+\hat{b} & =i \\
\hat{x}+\hat{c}+\hat{d} & =i \\
\hat{x}+\hat{e}+\hat{f} & =j \\
\hat{a}+\hat{c} & =\hat{2 e} \\
\hat{b}+\hat{d} & =\hat{2 f} .
\end{aligned}
$$


It follows that $i=j$ which cannot happen.

On the other hand, if $G$ is a cyclic group of order $n$, then depending on how $W$ was chosen, without loss of generality, we can assume that we have the following five cases to consider:

case 1:

$$
\begin{aligned}
\sigma(x)+a+b & =6 i \\
\sigma(x)+c+d & =6 i \\
\sigma(x)+e+f & =6 j \\
a+c & =2 e \\
b+d & =2 f
\end{aligned}
$$

This implies that $i=j$ since $3 \nmid|G|$ which cannot happen.

The remaining four cases are:

case 2 :

$$
\begin{aligned}
x+a+\sigma(b) & =6 i \\
x+c+\sigma(d) & =6 i \\
x+e+\sigma(f) & =6 j \\
a+c & =2 e \\
b+d & =2 f
\end{aligned}
$$

case 3 :

$$
\begin{aligned}
x+a+\sigma(b) & =6 i \\
x+c+\sigma(d) & =6 i \\
x+\sigma(e)+f & =6 j \\
a+c & =2 e \\
b+d & =2 f
\end{aligned}
$$

case 4:

$$
\begin{aligned}
x+a+\sigma(b) & =6 i \\
x+c+\sigma(d) & =6 i \\
\sigma(x)+e+f & =6 j \\
a+c & =2 e \\
b+d & =2 f
\end{aligned}
$$

case 5:

$$
\begin{aligned}
\sigma(x)+a+b & =6 i \\
\sigma(x)+c+d & =6 i \\
x+e+\sigma(f) & =6 j \\
a+c & =2 e \\
b+d & =2 f
\end{aligned}
$$


By relabeling $x, a, b, c, d, e, f$ as necessary, each of the above four sets of equations from cases 2 to 5 imply the following equation:

$$
\pm(\sigma(a)-a)+( \pm(\sigma(b)-b) \pm(\sigma(c)-c)) / 2=6(i-j)
$$

Since $\sigma$ swaps 0 with 2 and 4 with 6 , it is clear that each $\sigma(*)-*$ in Equation 3 can only take on values of 0 or \pm 2 . Thus Equation 3 implies that $\{0, \pm 1, \pm 2, \pm 3, \pm 4\} \cap\{6,12\} \neq \emptyset$. This cannot happen since $n \geq 17$.

So $\left((\mathbb{Z} / n \mathbb{Z} \times G) \cup\left\{\infty_{1}, \infty_{2}\right\}, B\right)$ is a 5 -sparse Steiner triple system. To see that it is average-free, project the triples of the system to their form. Then it is clear that only triples of type 1, 6 or 7 have the potential for being average triples. Triples of type 1 cannot be average triples since projecting to their subscripts would yield an average triple in $S$ which cannot happen. Triples of type 7 cannot be average triples by hypothesis. So assume that we have an average triple of type 6 . It follows that the form of the triple is an average triple in $T$. Thus the triple looks like $\left\{X_{a}, Y_{b}, Z_{c}\right\}$ where $2 Z=X+Y$. If $G=\mathbb{Z} / n \mathbb{Z}$, then the subscripts satisfy:

$$
\begin{aligned}
a+b+\sigma(c) & =6 i \\
a+b & =2 c
\end{aligned}
$$

where $i \in\{0,1,2\}$. Then $\sigma(c)+2 c \in\{0,6,12\}$. It follows that $\sigma(c) \neq c$ and so $c \in\{0,2,4,6\}$. Then $(\sigma(c)+2 c) \in\{2,4,14,16\}$ which is disjoint from $\{0,6,12\}$, a contradiction.

We arrive at a similar contradiction if we assume $G=H \times \mathbb{Z} / k \mathbb{Z}$ as in Definition 5.4. Projecting the elements of $G$ to $H$ (denoted by the $\sim$ operator), it is clear that the following equations must hold:

$$
\begin{aligned}
\tilde{a}+\tilde{b}+r & =2 \tilde{c} \\
\tilde{a}+\tilde{b} & =2 \tilde{c}
\end{aligned}
$$

which cannot be satisfied since $r$ is a non-zero element of $H$. Thus $((\mathbb{Z} / n \mathbb{Z} \times G) \cup$ $\left.\left\{\infty_{1}, \infty_{2}\right\}, B\right)$ is an average-free 5-sparse Steiner triple system.

To show that the earlier construction can contribute to providing orders that admit 5-sparse Steiner triple systems, we show the existence of an infinite class (of positive arithmetic density) of 5-sparse average-free systems.

Lemma 5.6. There exists 5-sparse average-free Steiner triple systems of order 9 n for odd $n, 7 \nmid n, n \geq 9$.

Proof. Recall in the proof of Lemma 4.3 we showed the existence of three 4 -sparse superdisjoint Steiner triple systems of order $3 n$ provided $7 \nmid n, n$ odd, $n \geq 9$. This gives rise to a 5 -sparse Steiner triple systems of order $9 n,(\mathbb{Z} / 3 \mathbb{Z} \times \mathbb{Z} / 3 \mathbb{Z} \times \mathbb{Z} / n \mathbb{Z}, B)$. The triples of $B$ are: 
1. $\left\{0_{0_{x}}, 0_{0_{y}}, 1_{1_{(x+y) / 2+a_{0}}}\right\},\left\{0_{0_{x}}, 1_{0_{y}}, 0_{1_{(x+y) / 2+a_{0}}}\right\},\left\{0_{1_{x}}, 0_{1_{y}}, 1_{2_{(x+y) / 2+a_{1}}}\right\}$, $\left\{0_{1_{x}}, 1_{1_{y}}, 0_{2_{(x+y) / 2+a_{1}}}\right\},\left\{0_{2_{x}}, 0_{2_{y}}, 1_{0_{(x+y) / 2+a_{2}}}\right\},\left\{0_{2_{x}}, 1_{2_{y}}, 0_{0_{(x+y) / 2+a_{2}}}\right\}$

2. $\left\{1_{0_{x}}, 1_{0_{y}}, 2_{1_{(x+y) / 2+b_{0}}}\right\},\left\{1_{0_{x}}, 2_{0_{y}}, 1_{1_{(x+y) / 2+b_{0}}}\right\},\left\{1_{1_{x}}, 1_{1_{y}}, 2_{2_{(x+y) / 2+b_{1}}}\right\}$, $\left\{1_{1_{x}}, 2_{1_{y}}, 1_{2_{(x+y) / 2+b_{1}}}\right\},\left\{1_{2_{x}}, 1_{2_{y}}, 2_{0_{(x+y) / 2+b_{2}}}\right\},\left\{1_{2_{x}}, 2_{2_{y}}, 1_{0_{(x+y) / 2+b_{2}}}\right\}$

3. $\left\{2_{1_{x}}, 2_{1_{y}}, 0_{0_{(x+y) / 2+c_{0}}}\right\},\left\{2_{1_{x}}, 0_{1_{y}}, 2_{0_{(x+y) / 2+c_{0}}}\right\},\left\{2_{2_{x}}, 2_{2_{y}}, 0_{1_{(x+y) / 2+c_{1}}}\right\}$, $\left\{2_{2_{x}}, 0_{2_{y}}, 2_{1_{(x+y) / 2+c_{1}}}\right\},\left\{2_{0_{x}}, 2_{0_{y}}, 0_{2_{(x+y) / 2+c_{2}}}\right\},\left\{2_{0_{x}}, 0_{0_{y}}, 2_{2_{(x+y) / 2+c_{2}}}\right\}$

4. $\left\{0_{0_{x}}, 1_{0_{x}}, 2_{0_{x}}\right\}$

5. $\left\{0_{1_{x}}, 1_{1_{x}}, 2_{1_{x}}\right\}$

6. $\left\{0_{2_{x}}, 1_{2_{x}}, 2_{2_{x}}\right\}$

7. $\left\{0_{1_{x+a_{0}}}, 0_{2_{x+a_{0}+a_{1}}}, 1_{0_{x}}\right\}$

8. $\left\{0_{0_{x}}, 0_{2_{x+a_{0}+a_{1}}}, 1_{1_{x+a_{0}}}\right\}$

9. $\left\{0_{0_{x}}, 0_{1_{x+a_{0}}}, 1_{2_{x+a_{0}+a_{1}}}\right\}$

10. $\left\{1_{1_{x+b_{0}}}, 1_{2_{x+b_{0}+b_{1}}}, 2_{0_{x}}\right\}$

11. $\left\{1_{0_{x}}, 1_{2_{x+b_{0}+b_{1}}}, 2_{1_{x+b_{0}}}\right\}$

12. $\left\{1_{0_{x}}, 1_{1_{x+b_{0}}}, 2_{2_{x+b_{0}+b_{1}}}\right\}$

13. $\left\{2_{1_{x+c_{1}}}, 2_{2_{x}}, 0_{0_{x+c_{0}+c_{1}}}\right\}$

14. $\left\{2_{0_{x+c_{0}+c_{1}}}, 2_{2_{x}}, 0_{1_{x+c_{1}}}\right\}$

15. $\left\{2_{0_{x+c_{0}+c_{1}}}, 2_{1_{x+c_{1}}}, 0_{2_{x}}\right\}$

for $x, y$ distinct elements of $\mathbb{Z} / n \mathbb{Z}$ and $a_{0}, a_{1}, a_{2}, b_{0}, b_{1}, b_{2}$ and $c_{0}, c_{1}, c_{2}$ are elements in $\mathbb{Z} / n \mathbb{Z}$ that satisfy the following conditions which we label as $(\dagger)$ :

$$
\begin{gathered}
a_{0}+a_{1}+a_{2}=0 \\
b_{0}+b_{1}+b_{2}=0 \\
c_{i}=\left(-a_{i}-b_{i}\right) / 2 \\
c_{i} \neq a_{j}+b_{k} \text { and } a_{i} \neq b_{i}
\end{gathered}
$$

where $i, j$ and $k$ are distinct. The exact values of these elements will be determined later.

We place a copy of the Steiner triple system $B$ on $\left(\mathbb{Z} /(9 n-2) \mathbb{Z} \cup\left\{\infty_{1}, \infty_{2}\right\}, C\right)$ by mapping the triples of our system via the following permutation

$$
\sigma: \mathbb{Z} / 3 \mathbb{Z} \times \mathbb{Z} / 3 \mathbb{Z} \times \mathbb{Z} / n \mathbb{Z} \rightarrow \mathbb{Z} /(9 n-2) \mathbb{Z} \cup\left\{\infty_{1}, \infty_{2}\right\}
$$


where

$$
\begin{aligned}
& \sigma\left(0_{0_{x}}\right)=0 n+x \\
& \sigma\left(2_{0_{x}}\right)=1 n+x \\
& \sigma\left(1_{1_{x}}\right)=2 n+x \\
& \sigma\left(0_{2_{x}}\right)=3 n+x \\
& \sigma\left(2_{2_{x}}\right)=4 n+x \\
& \sigma\left(1_{0_{x}}\right)=5 n+x \\
& \sigma\left(0_{1_{x}}\right)=6 n+x \\
& \sigma\left(2_{1_{x}}\right)=7 n+x \\
& \sigma\left(1_{2_{x}}\right)=8 n+x
\end{aligned}
$$

where $x \in \mathbb{Z} / n \mathbb{Z}$ except for the last case where $x \in \mathbb{Z} / n \mathbb{Z} \backslash\{n-2, n-1\}$. Lastly, assign $\sigma\left(1_{2_{n-2}}\right)=\infty_{1}$ and $\sigma\left(1_{2_{n-1}}\right)=\infty_{2}$. Similar to previous constructions, for elements $X_{Y_{x}} \in \mathbb{Z} / 3 \mathbb{Z} \times \mathbb{Z} / 3 \mathbb{Z} \times \mathbb{Z} / n \mathbb{Z}$, we define the form to be $X_{Y}$ and the subscript $x$.

It is easy to check that of the triples of $B$ of type 1,2 or 3, the only triples that may lead to average triples in $C$ when applying $\sigma$ are triples that look like:

$$
\begin{aligned}
\left\{0_{0_{0}}, 1_{0_{0}}, 0_{1_{-1}}\right\},\left\{0_{1_{-1}}, 1_{1_{0}}, 0_{2_{0}}\right\},\left\{1_{0_{-1}}, 2_{0_{0}}, 1_{1_{0}}\right\},\left\{2_{0_{0}}, 0_{0_{0}}, 2_{2_{-1}}\right\}, \\
\left\{2_{1_{-1}}, 0_{1_{0}}, 2_{0_{0}}\right\},\left\{2_{2_{0}}, 0_{2_{0}}, 2_{1_{-1}}\right\},\left\{2_{1_{0}}, 2_{1_{-1}}, 0_{0_{0}}\right\} .
\end{aligned}
$$

Then $a_{0}=-1, a_{1}=1 / 2, b_{0}=1 / 2, c_{2}=-1, c_{0}=1 / 2, c_{1}=-1$, or $c_{0}=1 / 2$, respectively. Further restrictions on the $a_{i}$ 's, $b_{i}$ 's and $c_{i}$ 's arise through analysis of triples of $B$ of type 4 through 15 . For triples of type 4,5 or 6 , the only triples that can lead to average triples in $C$ are triples that look like respectively:

$$
\begin{aligned}
& \left\{0_{0_{x}}, 2_{0_{y}}, 1_{0_{(x+y) / 2-1}}\right\} \\
& \left\{0_{1_{x}}, 2_{1_{y}}, 1_{1_{(x+y) / 2+1}}\right\} \\
& \left\{0_{2_{x}}, 2_{2_{y}}, 1_{0_{(x+y) / 2-1}}\right\}
\end{aligned}
$$

for some $x, y \in \mathbb{Z} / n \mathbb{Z}$. Since the subscripts of the triples are identical, each of the above triples cannot exist. For the triples of type 7 through 15, if an average triple arises from any of these types, then the following must hold for that type, respectively:

7. $\left\{0_{1_{x}}, 0_{2_{y}}, 1_{0_{(x+y) / 2}}\right\}$

8. $\left\{0_{0_{x}}, 0_{2_{y}}, 1_{1_{(x+y) / 2}}\right\}$

9. $\left\{0_{0_{x}}, 0_{1_{y}}, 1_{2_{(x+y) / 2-1}}\right\}$

10. $\left\{1_{1_{x}}, 1_{2_{y}}, 2_{0_{(x+y) / 2+1}}\right\}$

11. $\left\{1_{0_{x}}, 1_{2_{y}}, 2_{1_{(x+y) / 2}}\right\}$ 
12. $\left\{1_{0_{x}}, 1_{1_{y}}, 2_{2_{(x+y) / 2}}\right\}$

13. $\left\{2_{1_{x}}, 0_{0_{y}}, 2_{2_{(x+y) / 2}}\right\}$ or $\left\{2_{2_{x}}, 0_{0_{y}}, 2_{1_{(x+y) / 2-1}}\right\}$

14. $\left\{2_{2_{x}}, 0_{1_{y}}, 2_{0_{(x+y) / 2+1}}\right\}$ or $\left\{2_{0_{x}}, 0_{1_{y}}, 2_{2_{(x+y) / 2}}\right\}$

15. $\left\{2_{0_{x}}, 0_{2_{y}}, 2_{1_{(x+y) / 2-1}}\right\}$ or $\left\{2_{1_{x}}, 0_{2_{y}}, 2_{0_{(x+y) / 2+1}}\right\}$.

It follows that we have the following restrictions for the $a_{i}{ }^{\prime}$ s, $b_{i}$ 's and $c_{i}$ 's from each of the above triples, respectively:

7. $a_{1}=-2 a_{0}$

8. $a_{1}=a_{0}$

9. $a_{1}=-a_{0} / 2-1$

10. $b_{1}=-2 b_{0}-1$

11. $b_{1}=b_{0}$

12. $b_{1}=-b_{0} / 2$

13. $c_{1}=-c_{0} / 2$ or $c_{1}=c_{0}-2$

14. $c_{1}=-2 c_{0}+2$ or $c_{1}=-c_{0} / 2$

15. $c_{1}=c_{0}-2$ or $c_{1}=-2 c_{0}+2$

So, in summary, the triples of $C$ are average-free provided that the $a_{i}$ 's, $b_{i}$ 's and $c_{i}$ 's satisfy $(\dagger)$ as well as:

$$
\begin{array}{r}
a_{0} \neq-1 \\
b_{0} \neq 1 / 2 \\
c_{0} \neq 1 / 2 \\
c_{1} \neq-1 \\
a_{1} \notin\left\{1 / 2, a_{0},-2 a_{0},-a_{0} / 2-1\right\} \\
b_{1} \notin\left\{b_{0},-2 b_{0}-1,-b_{0} / 2\right\} \\
c_{1} \notin\left\{-1,1-c_{0},-c_{0} / 2, c_{0}-2,-2 c_{0}+2\right\}
\end{array}
$$

if $n \geq 9,7 \nmid n, n \neq 13$, set $a_{0}=0, a_{1}=4, a_{2}=-4, b_{0}=2, b_{1}=1, b_{2}=-3, c_{0}=-1$, $c_{1}=-5 / 2$, and $c_{2}=7 / 2$. It is easy to check that the above conditions are satisfied. If $n=13$, then setting $a_{0}=0, a_{1}=2, a_{2}=-2, b_{0}=2, b_{1}=6, b_{2}=-8, c_{0}=-1, c_{1}=-4$, and $c_{2}=5$ works.

Thus $\left(\mathbb{Z} /(9 n-2) \mathbb{Z} \cup\left\{\infty_{1}, \infty_{2}\right\}, C\right)$ is a 5 -sparse average-free Steiner triple system of order $9 n$. 


\section{Spectrum Of 5-sparse Steiner Triple Systems}

In the following discussion, we define the set of counting numbers as $\mathbb{N}=\{1,2,3, \ldots\}$.

Definition 6.1. Let $S$ and $T$ be two subsets of $\mathbb{N}$. Define the arithmetic density of $S$ as:

$$
d(S)=\lim _{n \rightarrow \infty} \frac{|\{x \in S: x \leq n\}|}{n} .
$$

Define the arithmetic density of $S$ as compared to $T$ as:

$$
d(S ; T)=\lim _{n \rightarrow \infty} \frac{|\{x \in S \cap T: x \leq n\}|}{|\{x \in T: x \leq n\}|} .
$$

Definition 6.2. Let $S, T \subseteq \mathbb{N}$. We say that $S$ has almost all the elements of $T$ if $d(S ; T)=1$.

Lemma 6.3. The arithmetic density of orders of meager systems is 1 as compared to the set of odd numbers.

Proof. By Lemma 3.6, using the fact that a 4-sparse Steiner triple system exists of order $n$ for any $n \equiv 1,3 \bmod 6$ except for $n=7,13$, we can find a meager system of order $r$ if $r=m n+2$ for some odd $m$ and $n$ with $n \geq 5, m \geq 7, m \neq 11,3 \nmid m$. This covers all odd $r$ except for $r$ of the form $p+2$ or $3 p+2$ for prime $p$ or $r \in\left\{27,57,77,123,167,365,3^{k}+\right.$ $\left.2,5\left(3^{k}\right)+2,11\left(3^{k}\right)+2: k \geq 0\right\}$. It is clear that the exceptions form a 0 -dense set. Thus the density of orders of meager systems is 1 as compared to the set of odd numbers.

Thus, we get the following Corollary:

Corollary 6.4. There exists a 5-sparse Steiner triple system of order $n$ for almost all $n \equiv 3 \bmod 6$.

For showing that there are 5-sparse Steiner triple systems that admit almost all orders $n$ for $n \equiv 1 \bmod 6$, we need to introduce the following lemmas:

Lemma 6.5. Let $a$ and $b$ be two relatively prime positive integers. Define the set $T$ as:

$$
T=\{p: p \text { is a prime number } \equiv a \bmod b\} .
$$

Then the set $W$ defined as

$$
W=\{x \in \mathbb{N}: p \mid x \text { for some } p \in T\}
$$

has arithmetic density 1.

Proof. Using the principle of inclusion/exclusion it is clear that the density of $W$ is given by:

$$
d(W)=1-\prod_{p \in T}\left(1-\frac{1}{p}\right) .
$$


Thus the density of $\bar{W}$, the complement of $W$ is:

$$
d(\bar{W})=\prod_{p \in T}\left(1-\frac{1}{p}\right)
$$

Moreover the reciprocal of this product is:

$$
\prod_{p \in T} \frac{1}{\left(1-\frac{1}{p}\right)}=\prod_{p \in T}\left(1+\frac{1}{p}+\frac{1}{p^{2}}+\frac{1}{p^{3}}+\ldots\right)>\sum_{p \in T} \frac{1}{p}
$$

It is well-known that $\sum_{p \in T} \frac{1}{p}=\infty(\operatorname{cf}[9])$. Thus $\prod_{p \in T}\left(1-\frac{1}{p}\right)=0$ and so $d(\bar{W})=0$ which implies that $d(W)=1$.

Lemma 6.6. Let $a$ and $b$ be two relatively prime positive integers. Define the set $T$ as:

$$
T=\{p: p \text { is a prime number } \equiv a \bmod b\} .
$$

Suppose we have a set $S$ with arithmetic density $r$ for some $r \in[0,1]$. Then the set $W \subseteq S$ where:

$$
W=\{x \in S: p \mid x \text { for some } p \in T\}
$$

has arithmetic density $r$ as well.

Proof. Consider the set $Q=S \backslash W$. Then

$$
Q=\{x \in S: \text { the prime factorization of } x \text { contains no primes of } T\} \text {. }
$$

It follows that $Q \subseteq\{x \in \mathbb{N}$ : the prime factorization of $x$ contains no primes of $T\}$ which by Lemma 6.5 has arithmetic density 0 . Thus $d(Q)=0$ and so $d(W)=r$.

Lemma 6.7. The arithmetic density of 5-sparse Steiner triple systems of orders $n \equiv 1$ $\bmod 6$ as compared to the set of numbers $n \in \mathbb{N}$ with $n \equiv 1 \bmod 6$ is 1 .

Proof. Define $P$ as the set of all primes $\equiv 17 \bmod 54$ and $R$ as the set of all primes $\equiv 25$ mod 126. Consider the set $W$ defined as:

$$
W=\left\{x \in \mathbb{N}: x \equiv 5 \bmod 6, p_{1} p_{2} \mid x \text { for some } p_{1} \in P \text { and } p_{2} \in R\right\}
$$

By applying Lemma 6.6 twice it is clear that $W$ has arithmetic density $1 / 6$.

Define $U$ as:

$$
U=\{x-2: x \equiv 3 \bmod 6 \text { and } x \text { admits a } 5 \text {-sparse Steiner triple system }\}
$$

and define $W^{\prime}$ as:

$$
W^{\prime}=\left\{p_{1} p_{2} u: p_{1} \in P, p_{2} \in R \text { and } u \in U\right\}
$$


Claim: $d\left(W^{\prime} ; W\right)=1$. The claim is clear since elements in $W$ can be written as $p_{1} p_{2} u$ where $p_{1} \in P, p_{2} \in R$ and $u \equiv 1 \bmod 6$, and restricting $u$ to lie in $U$ eliminates only a set of arithmetic density 0 by Corollary 6.4.

Thus $\mathrm{d}\left(\mathrm{W}^{\prime}\right)=1 / 6$. Next, define $W^{\prime \prime}=\left\{x+2: x \in W^{\prime}\right\}$. Then $d\left(W^{\prime \prime}\right)=1 / 6$. We will see that $W^{\prime \prime}$ consists of only numbers that admit 5-sparse Steiner triple systems: By Lemma 5.6, there exist average-free 5-sparse Steiner triple systems of order $9 n$ provided $n$ odd, $n \geq 9$ and $7 \nmid n$. Thus there exists average-free 5 -sparse Steiner triple systems of order $x+2$ for $x \in R$ with respect to the group $\mathbb{Z} / x \mathbb{Z}$. By Theorem 1.4 , there exists 5 -sparse Steiner triple systems of order $x+2$ for $x \in P$. Thus, by Lemma 5.5 we can produce average-free 5-sparse Steiner triple systems of order $p_{1} p_{2}+2$ for any $p_{1} \in P$ and $p_{2} \in R$ with respect to the group $\mathbb{Z} / p_{1} \mathbb{Z} \times \mathbb{Z} p_{2} \mathbb{Z}$. Again by Lemma 5.5 (average-free) 5 -sparse Steiner triple systems of order $p_{1} p_{2} x+2$ exist for any $p_{1} \in P, p_{2} \in R$ and $x \in U$ (with respect to the group $\mathbb{Z} / p_{1} \mathbb{Z} \times \mathbb{Z} p_{2} \mathbb{Z} \times \mathbb{Z} / x \mathbb{Z}$ ). Hence $W^{\prime \prime}$ consists of only orders that admit 5-sparse Steiner triple systems. Since $W^{\prime \prime}$ consists of only numbers $n$ where $n \equiv 1$ $\bmod 6$, it is clear that the arithmetic density of $W^{\prime \prime}$ as compared to all numbers congruent to $1 \bmod 6$ is 1 , and so the arithmetic density of 5 -sparse Steiner triple systems of orders $n \equiv 1 \bmod 6$ as compared to the set of numbers $n \in \mathbb{N}$ with $n \equiv 1 \bmod 6$ is 1 .

Combining Lemma 6.7 and Corollary 6.4 gives us:

Theorem 6.8. The arithmetic density of the spectrum of 5-sparse Steiner triple systems as compared to the set of admissible orders is 1 .

\section{Summary of Methods}

In showing that almost all admissible orders admit a 5-sparse Steiner triple system, we implemented various constructions:

- In section 3, we constructed meager systems of order $m n+2$ for most $m, n$ by Lemma 3.6. A meager system is realized for almsot all odd numbers by this construction which, in turn, allows us to construct 5-sparse Steiner triple systems of order $v$ for almost all admissible $v$ such that $v \equiv 3 \bmod 6$ as in Corollary 6.4.

- In section 4, we constructed a special class of super-disjoint Steiner triple systems of order $3 n$ for large odd $n$ where $7 \nmid n$ by Lemma 4.3. This gave rise to a 5 -sparse Steiner triple system of order $9 n$.

- In section 5, we considered a recursive construction for creating average-free 5-sparse Steiner triple system of order $m n+2$ from an average-free 5-sparse Steienr triple system of order $m+2$ and a 5 -sparse Steiner triple system of order $n+2$ as described in Lemma 5.5.

Manipulating the 5-sparse Steiner triple system of order $9 n$ from section 4, we showed that such a system can be made to be average-free as described in Lemma 5.6. 
- In section 6, we used the average-free construction in Lemma 5.5 on the averagefree Steiner triple systems of order $9 n$ and on another set of 5-sparse Steiner triple sytems (of positive arithmetic density) given by Theorem 1.4 to produce a plethora of average-free 5-sparse Steiner triple systems. Applying Lemma 5.5 once again on this new set of average-free 5-sparse Steiner triple systems and on the dense set of 5 -sparse Steiner triple sytems of order $v$ with $v \equiv 3 \bmod 6$ gives us 5 -sparse Steiner triple systems of order $v$ for almost all $v \equiv 1 \bmod 6$ as described in Lemma 6.7. And so, 5-sparse Steiner triple systems exist for almost all admissible $v$.

\section{Appendix: Proof of Lemma 3.6}

To aid in the proof of Lemma 3.6, we utilize the following lemma:

Lemma 8.1. Let $V$ be an $n$-set and let $V_{0}, V_{1}$ and $V_{2}$ be subsets of $V$. Suppose we have three pairwise really disjoint $N_{2}$ deleted symmetric squares on $\left(V, B_{i}\right)$ with subsquares $\left(V_{i}, M_{i}\right)$ for $i \in \mathbb{Z} / 3 \mathbb{Z}$ such that every triple of $B_{i} \backslash M_{i}$ is super-symmetric and $B_{i} \cap \mathcal{P}_{3}\left(V_{j}\right)=\emptyset$ for $i \neq j$. Then $\left(V, B_{0}, B_{1}, B_{2}\right)$ forms a meager system if the system avoids $Q$ configurations:

$$
(x, y, z) \in B_{i},(x, z, w) \in B_{i+1}, \text { and }(x, w, y) \in B_{i+2}
$$

and a type of $M_{3}$ configuration:

$$
(x, y, z),(y, w, x) \in B_{i} \cap M_{i},(x, z, v) \in B_{i+1}, \text { and }(x, v, w) \in B_{i+2} .
$$

Proof. Suppose we have such systems as described in the hypothesis. By assumption, we already know that the system $\left(V, B_{0}, B_{1}, B_{2}\right)$ avoids $Q$ configurations. Also, if there is an $M_{3}$ configuration, it must look like:

$$
(x, y, z),(y, w, x) \in B_{i} \backslash M_{i},(x, z, v) \in B_{i+1} \text { and }(x, v, w) \in B_{i+2} .
$$

Then triples $(x, y, z)$ and $(y, w, x)$ are super-symmetric in $B_{i}$. Thus $w=z$. However, this contradicts $B_{i+1}$ and $B_{i+2}$ being really disjoint. Also, by super-disjointness, the systems cannot contain any $M_{1}$ configurations. Lastly, suppose the systems contained an $M_{2}$ configuration, say,

$$
(x, y, z),(y, v, x),(x, v, w) \in B_{i},(z, w, x) \in B_{i+1} .
$$

If any of the first three triples are super-symmetric in $B_{i}$, then so are the other two. Then $v=w$ which cannot happen since $B_{i}$ has a removed idempotent diagonal. Thus, the first three triples of the $M_{2}$ configuration are non super-symmetric triples of $B_{i}$. It follows that $\{x, v, w\} \subseteq V_{i}$ which cannot happen. Hence the system is a meager system.

We are now ready to prove Lemma 3.6: 
Proof of Lemma 3.6. Let $m$ be an odd number, $m>1, m \equiv 1,5 \bmod 6$ with $m \neq 5,11$. Let $V$ be the $m$-set containing three distinct points labeled as $t, t^{\prime}, t^{\prime \prime}$, and not containing the two points: $\infty_{1}$ and $\infty_{2}$. Let $n \geq 5$ be an odd number. Define an $n$-set $N$ as $=$ $\mathbb{Z} /((n+2) \mathbb{Z}) \backslash\{n, n+1\}$. Set

$$
\begin{aligned}
& V_{0}=\left\{t_{x} \mid x \in N \cup\left\{\infty_{1}, \infty_{2}\right\}\right\} \\
& V_{1}=\left\{t_{x}^{\prime} \mid x \in N \cup\left\{\infty_{1}, \infty_{2}\right\}\right\} \\
& V_{2}=\left\{t_{x}^{\prime \prime} \mid x \in N \cup\left\{\infty_{1}, \infty_{2}\right\}\right\}
\end{aligned}
$$

Define $\mathcal{S}$ as:

$$
\mathcal{S}=\{(x, y, z) \mid 2 z=x+y, x, y, z \in \mathbb{Z} /((n+2) \mathbb{Z})\} .
$$

Define $S_{i}$ on $V_{i}$ as a copy of $S$ by mapping $x$ to $t_{x}, t_{x}^{\prime}$ or $t_{x}^{\prime \prime}$, respectively for $i \in \mathbb{Z} / 3 \mathbb{Z}$ for each $x \in N$, and mapping $n \mapsto \infty_{1}$ and $n+1 \mapsto \infty_{2}$.

Put a group structure on $N$ by identifying the elements in some way with $\mathbb{Z} / n \mathbb{Z}$. So as to not confuse this structure with the structure of $\mathbb{Z} /((n+2) \mathbb{Z})$, define the addition operator of this group as $\hat{+}$. Also, operators such as the minus operator, - , or multiplication/division that are used in an equation that involves the $\hat{+}$ operator should be taken as the usual meaning with respect to this group.

For $i \in\{0,1,2\}$, define $L^{i}$ on $N$ as the $N_{2}$ Latin squares:

$$
L^{i}=\{(x, y, z) \mid x \hat{+} y \hat{+} z=i\}
$$

Choose $R^{0}$ as an idempotent Valek square of order $n$ on $N$. Let $R^{1}$ be the Valek square of order $n$ obtained by "shifting" the entries of $R^{0}$ by 1 , i.e. defining $R^{1}=$ $\left\{(x, y, z \hat{+} 1) \mid(x, y, z) \in R^{0}\right\}$. Similarly, let $R^{2}$ be the Valek square of order $n$ obtained by shifting the entries of $R^{0}$ by 2 . The point of this is to have three pairwise disjoint Valek squares.

We construct three deleted symmetric squares on $\mathcal{V}=(V \times N) \cup\left\{\infty_{1}, \infty_{2}\right\}$. To aid in the construction let us define $\operatorname{Pr}: \mathcal{V} \rightarrow V \cup\left\{\infty_{1}, \infty_{2}\right\}$ by mapping the element $x_{a}$ to its form $x$ and by mapping $\infty_{1}$ to $\infty_{1}$ and $\infty_{2}$ to $\infty_{2}$. By convention we define the form of $\infty_{1}$ to be $\infty_{1}$ and the form of $\infty_{2}$ to be $\infty_{2}$. The construction is broken up into two cases: $m=7$ and $m>7$ :

case 1: $m=7$. Let the 7 -set $V$ be written as $V=\left\{0,1,2,3, t, t^{\prime}, t^{\prime \prime}\right\}$. Let $T_{0}, T_{1}$ and $T_{2}$ be the following three triple sets of 4-sparse Steiner triple systems of order 9 on $V \cup\left\{\infty_{1}, \infty_{2}\right\}$ :

$$
\begin{aligned}
T_{0}= & \left\{\left\{t, 1, t^{\prime}\right\},\left\{2, t^{\prime}, 0\right\},\left\{2, t, t^{\prime \prime}\right\},\left\{3,1, t^{\prime \prime}\right\},\{3, t, 0\},\left\{\infty_{1}, t^{\prime}, t^{\prime \prime}\right\},\right. \\
& \left.\left\{\infty_{1}, 1,0\right\},\left\{\infty_{1}, 3,2\right\},\left\{\infty_{2}, 0, t^{\prime \prime}\right\},\left\{\infty_{2}, 2,1\right\},\left\{\infty_{2}, 3, t^{\prime}\right\},\left\{\infty_{2}, \infty_{1}, t\right\}\right\} \\
T_{1}= & \left\{\left\{t, t^{\prime}, 0\right\},\left\{2,0, t^{\prime \prime}\right\},\left\{2,1, t^{\prime}\right\},\left\{3, t^{\prime}, t^{\prime \prime}\right\},\{3, t, 1\},\left\{\infty_{1}, 1, t^{\prime \prime}\right\},\right. \\
& \left.\left\{\infty_{1}, 2, t\right\},\left\{\infty_{1}, 3,0\right\},\left\{\infty_{2}, 1,0\right\},\left\{\infty_{2}, t, t^{\prime \prime}\right\},\left\{\infty_{2}, 3,2\right\},\left\{\infty_{2}, \infty_{1}, t^{\prime}\right\}\right\} \\
T_{2}= & \left\{\left\{1,0, t^{\prime \prime}\right\},\left\{t, t^{\prime}, t^{\prime \prime}\right\},\{2, t, 0\},\left\{3,1, t^{\prime}\right\},\left\{3,2, t^{\prime \prime}\right\},\left\{\infty_{1}, t, 1\right\},\right. \\
& \left.\left\{\infty_{1}, 2, t^{\prime}\right\},\left\{\infty_{1}, 3,0\right\},\left\{\infty_{2}, t^{\prime}, 0\right\},\left\{\infty_{2}, 2,1\right\},\left\{\infty_{2}, 3, t\right\},\left\{\infty_{2}, \infty_{1}, t^{\prime \prime}\right\}\right\}
\end{aligned}
$$


For $i \in \mathbb{Z} / 3 \mathbb{Z}$, define $\Omega_{i}$ as the following sets of ordered pairs from $V$ :

$$
\begin{aligned}
& \Omega_{0}=(0,1),(1,2),(2,3),\left(3, t^{\prime}\right),\left(t^{\prime}, t^{\prime \prime}\right),\left(t^{\prime \prime}, 0\right) \\
& \Omega_{1}=(0,1),\left(1, t^{\prime \prime}\right),\left(t^{\prime \prime}, t\right),(t, 2),(2,3),(3,0) \\
& \Omega_{2}=\left(0, t^{\prime}\right),(3,0),(t, 3),(1, t),(2,1),\left(t^{\prime}, 2\right)
\end{aligned}
$$

For each ordered pair in $(x, y) \in \Omega_{i}$, define the Valek squares $R_{\{x, y\}}^{i}$ as equal to $R^{i}$ except set $R_{\{0,1\}}^{1}=R_{\left\{1, t^{\prime \prime}\right\}}^{1}=R_{\left\{t, t^{\prime \prime}\right\}}^{1}=R^{0}$.

For each triple $\{x, y, z\} \in T_{i}$, choose an ordering $(x, y, z)$ and define $L_{x, y, z}^{i}=L^{i}$ except for the following cases:

$$
\begin{gathered}
L_{0,2, t^{\prime}}^{0}=L_{1, t^{\prime}, t}^{0}=L_{2, t, t^{\prime \prime}}^{0}=L_{3,1, t^{\prime \prime}}^{0}=\{(x, y, z) \mid z=2 x \hat{+} y, x, y, z \in \mathbb{Z} / n \mathbb{Z}\} \\
L_{t^{\prime}, t, 0}^{1}=L_{t, 3,1}^{1}=L_{t^{\prime \prime}, 0,2}^{1}=L_{t^{\prime \prime}, t, 3}^{1}=L_{t^{\prime \prime}, t^{\prime}, 3}^{1}=\{(x, y, z) \mid z=x \hat{+} y, x, y, z \in \mathbb{Z} / n \mathbb{Z}\} \\
L_{0, t, 2}^{2}=L_{1,3, t^{\prime}}^{2}=L^{0} \\
L_{0,1, t^{\prime \prime}}^{2}=\{(x, y, z) \mid 2 z=x \hat{+} y \hat{+} 1, x, y, z \in \mathbb{Z} / n \mathbb{Z}\} .
\end{gathered}
$$

Next, for each $i \in \mathbb{Z} / 3 \mathbb{Z}$, construct a deleted symmetric square on $\mathcal{V}=(V \times \mathbb{Z} / n \mathbb{Z}) \cup$ $\left\{\infty_{1}, \infty_{2}\right\}$ following the construction from Lemma 3.5 with the $T_{i}, S_{i}, \Omega_{i}, R_{*, *}^{i}$ and $L_{*, *, *}^{i}$ taking the role of $\mathcal{T}, \mathcal{S}, \Omega, R_{*, *}$ and $L_{*, *, *}$ of the lemma. Call the resulting squares $B_{i}$ for $i \in \mathbb{Z} / 3 \mathbb{Z}$. We must show that $\left(\mathcal{V}, B_{0}, B_{1}, B_{2}\right)$ is a meager system. Notice that the squares are really disjoint and furthermore, the forms of elements of triples of $S_{0}, S_{1}$ and $S_{2}$ are $\left\{\infty_{1}, \infty_{2}, t\right\},\left\{\infty_{1}, \infty_{2}, t^{\prime}\right\}$ and $\left\{\infty_{1}, \infty_{2}, t^{\prime \prime}\right\}$. By virtue of the construction, any ordered triple of elements from these last three sets only lie in $B_{0}, B_{1}$ and $B_{2}$, respectively. Furthermore, each $S_{i}$ forms the triples of a subsystem of $B_{i}$ and the triples in $B_{i} \backslash S_{i}$ are all super-symmetric in $B_{i}$. Thus the system $\left(\mathcal{V}, B_{0}, B_{1}, B_{2}\right)$ satisfy the conditions of Lemma 8.1. So all we must check is that the system avoids $M_{3}^{\prime}$ configurations and $Q$ configurations.

We have three cases to consider for checking whether any $M_{3}^{\prime}$ configurations:

$$
(x, y, z),(y, w, x) \in B_{i} \cap S_{i},(x, z, v) \in B_{i+1} \text {, and }(x, v, w) \in B_{i+2}
$$

exist in the system.

1. $i=0$. The forms of the elements $x, y, z, w \in\left\{t, \infty_{1}, \infty_{2}\right\}$. Then the form of $v$ is $t^{\prime \prime}$, and we arrive at the following $M_{3}^{\prime}$ configuration:

$$
\left(\infty_{2}, t_{0}, t_{-1 / 2}\right),\left(t_{0}, \infty_{1}, \infty_{2}\right) \in S_{0},\left(\infty_{2}, t_{-1 / 2}, t_{-3}^{\prime \prime}\right\} \in B_{1},\left(\infty_{2}, t_{-3}^{\prime \prime}, \infty_{1}\right) \in B_{2} .
$$

However, this cannot happen since the triple $\left(\infty_{2}, t_{-3}, t_{-1 / 2}^{\prime \prime}\right) \in B_{1}$ must have $-3=$ $-1 / 2$ which fails since $n+2 \neq 5$.

2. $i=1$. The forms of the elements $x, y, z, w \in\left\{t^{\prime}, \infty_{1}, \infty_{2}\right\}$. Then the form of $v$ is $t^{\prime \prime}$, and we arrive at the following $M_{3}^{\prime}$ configuration:

$$
\left(\infty_{1}, t_{0}^{\prime}, \infty_{2}\right),\left(t_{0}^{\prime}, t_{-4}^{\prime}, \infty_{1}\right) \in B_{1},,\left(\infty_{1}, \infty_{2}, t_{-3 / 2}^{\prime \prime}\right) \in B_{2},\left(\infty_{1}, t_{-3 / 2}^{\prime \prime}, t_{-4}^{\prime} \in B_{0} .\right.
$$

It follows that we require $-3 / 2=-4$ which cannot be satsified since $n+2 \neq 5$. 


\begin{tabular}{|l||ccc|}
\hline & $\operatorname{Pr}\left(B_{0}\right)$ & $\operatorname{Pr}\left(B_{1}\right)$ & $\operatorname{Pr}\left(B_{2}\right)$ \\
\hline$Q_{1}$ & $\left(\infty_{1}, t^{\prime \prime}, t^{\prime}\right)$ & $\left(\infty_{1}, t^{\prime}, \infty_{2}\right)$ & $\left(\infty_{1}, \infty_{2}, t^{\prime \prime}\right)$ \\
\hline$Q_{2}$ & $\left(\infty_{2}, \infty_{1}, t\right)$ & $\left(\infty_{2}, t, t^{\prime \prime}\right)$ & $\left(\infty_{2}, t^{\prime \prime}, \infty_{1}\right)$ \\
\hline$Q_{3}$ & $\left(0, t^{\prime \prime}, \infty_{2}\right)$ & $\left(0, \infty_{2}, 1\right)$ & $\left(0,1, t^{\prime \prime}\right)$ \\
\hline$Q_{4}$ & $\left(1,0, \infty_{1}\right)$ & $\left(1, \infty_{1}, t^{\prime \prime}\right)$ & $\left(1, t^{\prime \prime}, 0\right)$ \\
\hline$Q_{5}$ & $\left(1, t^{\prime}, t\right)$ & $(1, t, 3)$ & $\left(1,3, t^{\prime}\right)$ \\
\hline$Q_{6}$ & $\left(3,1, t^{\prime \prime}\right)$ & $\left(3, t^{\prime \prime}, t^{\prime}\right)$ & $\left(3, t^{\prime}, 1\right)$ \\
\hline$Q_{7}$ & $\left(0,2, t^{\prime}\right)$ & $\left(0, t^{\prime}, t\right)$ & $(0, t, 2)$ \\
\hline$Q_{8}$ & $\left(2, t, t^{\prime \prime}\right)$ & $\left(2, t^{\prime \prime}, 0\right)$ & $(2,0, t)$ \\
\hline
\end{tabular}

Table 1: Case $m=7$; Possible Forms for $Q$ Configurations

3. $i=2$. The forms of the elements $x, y, z, w \in\left\{t^{\prime \prime}, \infty_{1}, \infty_{2}\right\}$. Then $\operatorname{Pr}(v) \in\left\{t, t^{\prime}\right\}$. If $\operatorname{Pr}(v)=t$, we arrive at the following $M_{3}^{\prime}$ configuration:

$$
\left(\infty_{2}, t_{-3}^{\prime \prime}, \infty_{1}\right),\left(t_{-3}^{\prime \prime}, t_{1}^{\prime \prime} \infty_{2}\right) \in S_{2},\left(\infty_{2}, \infty_{1}, t_{-3 / 2}\right) \in B_{0},\left(\infty_{2}, t_{-3 / 2}, t_{1}^{\prime \prime}\right) \in B_{1} .
$$

Then we require $-3 / 2=1$ which is impossible since $n+2 \neq 5$. In the case where $\operatorname{Pr}(v)=t^{\prime}$, we arrive at the $M_{3}^{\prime}$ configuration:

$$
\left(\infty_{1}, t_{-3}^{\prime \prime}, t_{-5 / 2}^{\prime \prime}\right),\left(t_{-3}^{\prime \prime}, \infty_{2}, \infty_{1}\right) \in S_{2},\left(\infty_{1}, t_{-5 / 2}^{\prime \prime}, t_{0}^{\prime}\right) \in B_{0},\left(\infty_{1}, t_{0}^{\prime}, \infty_{2}\right) \in B_{1} .
$$

which cannot happen since $-5 / 2 \neq 0$.

Hence there are no $M_{3}^{\prime}$ configurations in the system.

Let us investigate whether there are any $Q$ configurations. Suppose there is a $Q$ configuration. Projecting the configuration to its form only yields the eight possibilities listed in Table 1. Because of how the assignments of subscripts were given, without loss of generality we only need to check what the subscripts are in the first five cases from the table:

$Q_{1}$ :

$$
\left(\infty_{1}, t_{-3 / 2}^{\prime \prime}, t_{0}^{\prime}\right) \in B_{0},\left(\infty_{1}, t_{0}^{\prime}, \infty_{2}\right) \in B_{1},\left(\infty_{1}, \infty_{2}, t_{-3 / 2}^{\prime \prime}\right) \in B_{2} .
$$

The subscripts of the forms $t^{\prime \prime}$ and $t$ in the triple form $\left(\infty_{1}, t^{\prime \prime}, t^{\prime}\right) \in \operatorname{Pr}\left(B_{0}\right)$ are derived from an idempotent Valek square, and so $-3 / 2=0$. However, this cannot happen since $n+2 \neq 3$.

$Q_{2}$ :

$$
\left(\infty_{2}, \infty_{1}, t_{-3 / 2}\right) \in B_{0},\left(\infty_{2}, t_{-3 / 2}, t_{-3}^{\prime \prime}\right) \in B_{1},\left(\infty_{2}, t_{-3}^{\prime \prime}, \infty_{1}\right) \in B_{2}
$$

The subscripts of the forms $t$ and $t^{\prime \prime}$ in the triple form $\left(\infty_{2}, t, t^{\prime \prime}\right) \in \operatorname{Pr}\left(B_{1}\right)$ are derived from an idempotent Valek square, and so $-3 / 2=-3$. However, this cannot happen since $n+2 \neq 3$. 
$Q_{3}:$

$$
\left(0_{x}, t_{x}^{\prime \prime}, \infty_{2}\right) \in B_{0},\left(0_{x}, \infty_{2}, 1_{x}\right) \in B_{1},\left(0_{x}, 1_{x}, t_{x}^{\prime \prime}\right) \in B_{2}
$$

for some $x$. This cannot be since the Latin square determining the subscripts of the triple form $\left(0,1, t^{\prime \prime}\right) \in \operatorname{Pr}\left(B_{2}\right)$ has the property that there are no triples $(x, x, x)$ for $x \in N$.

$Q_{4}:$

$$
\left(1_{x}, 0_{x}, \infty_{1}\right) \in B_{0},\left(1_{x}, \infty_{1}, t_{x}^{\prime \prime}\right) \in B_{1},\left(1_{x}, t_{x}^{\prime \prime}, 0_{x}\right) \in B_{2}
$$

for some $x$. This cannot happen for the same reason as in the previous case.

$Q_{5}:$

$$
\left(1_{x}, t_{y}^{\prime}, t_{2 x \hat{+} y}\right) \in B_{0},\left(1_{x}, t_{2 x \hat{+} y}, 3_{-x-y}\right) \in B_{1},\left(1_{x}, 3_{-x-y}, t_{y}^{\prime}\right) \in B_{2}
$$

for some $x$ and $y$. This cannot be since the Latin square determining the subscripts of the triple form $\left(1,3, t^{\prime}\right) \in \operatorname{Pr}\left(B_{2}\right)$ is $L^{2}$ which does not have the triple $(x, y,-x-y)$.

case 2: $m>7$. In this case we need to choose a 4-sparse Steiner triple system $\left(V \cup\left\{\infty_{1}, \infty_{2}\right\}, T_{0}\right)$ of order $m+2$ for the construction. To help simplify the number of configurations to check, we wish the Steiner triple system to have certain properties:

For any Steiner triple system of order $v$ with $v \geq 7$, it is always possible to find a subgraph that is a pre-mitre, i.e. has four distinct triples that look like $\{a, b, c\},\{a, f, g\}$, $\{a, d, e\}$ and $\{c, g, e\}$. Graphically, a pre-mitre looks like:

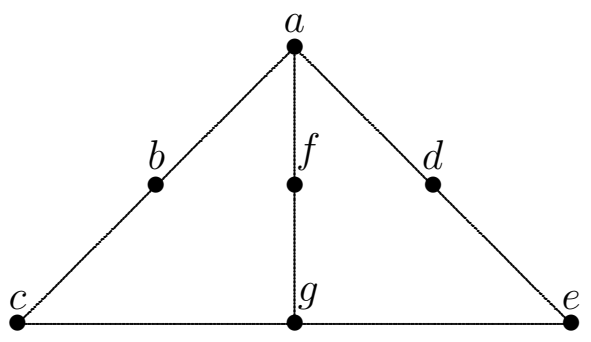

If $\{f, b, d\}$ is a triple of the system, then the pre-mitre can be completed to a mitre:

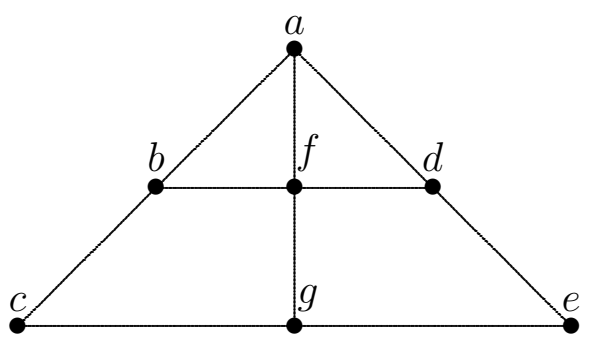

We wish to find a 4-sparse Steiner triple system that has a pre-mitre which does not complete to a mitre. Assmus in [1] showed that the only Steiner triple systems for which every pre-mitre completes to a mitre are those that are an affine space over $G F(3)$ as well as the Hall systems. The orders of these systems are exactly the powers of 3 . Thus to ensure that our 4-sparse Steiner triple system has a pre-mitre which does not complete to a mitre, we can choose 5 -sparse Steiner triple systems of order $m+2$ whenever $m+2$ 
is a power of $3, m \geq 81$ by Lemma 5.6 in Section 5 . When $m+2=27$, we can utilize a 5 -sparse Steiner triple system of order 27 developed from the meager system of order 9 , $\left(\mathbb{Z} / 9 \mathbb{Z}, K_{0}, K_{1}, K_{2}\right)$, where each deleted symmetric square $K_{i}$ is transitive with respect to $\mathbb{Z} / 9 \mathbb{Z}$ with starter blocks:

\begin{tabular}{|c|c|}
\hline square & starter blocks \\
\hline$K_{0}$ & $(1,7,0),(2,4,0),(3,8,0),(5,6,0)$ \\
\hline$K_{1}$ & $(1,5,0),(2,4,0),(3,6,0),(7,8,0)$ \\
\hline$K_{2}$ & $(1,3,0),(2,5,0),(4,8,0),(6,7,0)$ \\
\hline
\end{tabular}

Lastly, if $m+2=9$, the earlier case covererd this. So let us consider such a system and rearrange the points in such a way that $\left\{t, t^{\prime}, t^{\prime \prime}\right\},\left\{t, \infty_{1}, \infty_{2}\right\} \in T_{0}$ and the following pre-mitre:

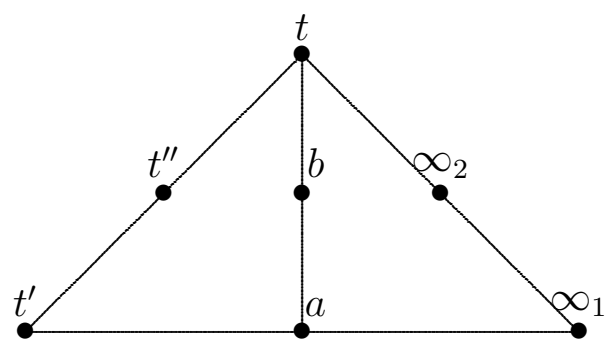

does not complete to a mitre.

Now define permutations $\sigma_{1}$ and $\sigma_{2}$ on $V \cup\left\{\infty_{1}, \infty_{2}\right\}$ as cycles where $\sigma_{1}$ is the cycle $\left(t, \infty_{1}, t^{\prime}, t^{\prime \prime}\right)$ and $\sigma_{2}$ is the cycle $\left(t, \infty_{2}, t^{\prime \prime}, t^{\prime}\right)$. Apply these permutations on $T_{0}$ to form triple sets $T_{1}$ and $T_{2}$, respectively.

Define $\{\alpha, \beta, \chi, \delta, \epsilon, \phi, \gamma, \eta, \iota, \kappa, \lambda\} \subseteq V \cup\left\{\infty_{1}, \infty_{2}\right\}$ such that the following triples are in $T_{0}$ :

$$
\begin{array}{r}
\left\{a, t^{\prime}, \infty_{1}\right\},\{\beta, \alpha, t\},\left\{\chi, t^{\prime \prime}, \infty_{2}\right\},\{\delta, t, \chi\},\left\{\epsilon, \chi, t^{\prime}\right\} \\
\left\{\phi, t^{\prime}, \infty_{2}\right\},\left\{\gamma, \phi, t^{\prime \prime}\right\},\left\{\eta, \alpha, t^{\prime \prime}\right\},\left\{\iota, t^{\prime \prime}, \infty_{1}\right\},\left\{\kappa, \iota, t^{\prime}\right\}\left\{\iota, \lambda, \infty_{2}\right\}
\end{array}
$$

Note that by the definitions of these elements and by the fact that $T_{0}$ has no Paschs we have $\{\alpha, \beta, \chi, \delta, \epsilon, \phi, \gamma, \eta, \iota, \kappa, \lambda\} \cap\left\{t, t^{\prime}, t^{\prime \prime}, \infty_{1}, \infty_{2}\right\}=\emptyset$. So, $\sigma_{1}$ and $\sigma_{2}$ do not move any of these elements.

Define $\Omega_{i}$ as in Lemma 3.5 such that $\left(t^{\prime}, f\right) \in \Omega_{0},(\beta, \alpha) \in \Omega_{1}$ and $(\delta, \chi) \in \Omega_{2}$.

For $\alpha \in \mathbb{Z} / 3 \mathbb{Z}$ and for each $(x, y) \in \Omega_{i}$ define $R_{\{x, y\}}^{i}=R^{i}$ except for:

$$
\begin{array}{r}
R_{\{\iota, \lambda\}}^{0}=R^{1} \\
R_{\{\alpha, \beta\}}^{1}=R_{\{\chi, t\}}^{1}=R_{\left\{t, t^{\prime \prime}\right\}}^{1}=R_{\{\iota, \lambda\}}^{1}=R^{0} \\
R_{\{\alpha, t\}}^{2}=R_{\{\chi, \delta\}}^{2}=R_{\left\{t, t^{\prime}\right\}}^{2}=R^{0} .
\end{array}
$$

For $i \in \mathbb{Z} / 3 \mathbb{Z}$ and for each triple $\{X, Y, Z\} \in T_{i}$ with $X, Y, Z \notin\left\{\infty_{1}, \infty_{2}\right\}$, choose an ordering for $\{X, Y, Z\}$ and define $L_{X, Y, Z}^{i}=L^{i}$ except for some of the following assignments for triples that intersect the set $\left\{t, t^{\prime}, t^{\prime \prime}\right\}$ : 
Define

$$
L_{t, t^{\prime}, t^{\prime \prime}}^{0}=\{(x, y, z) \mid z=(x \hat{+} y) / 2, x, y, z \in N\} .
$$

For triples $\{t, X, Y\} \in T_{0}$ with $\{X, Y\} \cap\left\{t, t^{\prime}, t^{\prime \prime}\right\}=\emptyset$ define

$$
L_{t, X, Y}^{0}=\{(x, y, z) \mid x=(y \hat{+} z) / 2 \hat{+} 1, x, y, z \in N\} .
$$

For triples $\left\{t^{\prime}, X, Y\right\} \in T_{0}$ with $\{X, Y\} \cap\left\{t, t^{\prime}, t^{\prime \prime}\right\}=\emptyset$ define

$$
L_{t^{\prime}, X, Y}^{0}=\{(x, y, z) \mid x=y \hat{+} z, x, y, z \in N\} .
$$

For triples $\left\{t^{\prime \prime}, X, Y\right\} \in T_{0}$ with $\{X, Y\} \cap\left\{t, t^{\prime}, t^{\prime \prime}\right\}=\emptyset$ define

$$
L_{t^{\prime \prime}, X, Y}^{0}=\{(x, y, z) \mid x=y \hat{+} z, x, y, z \in N\} .
$$

Also, define

$$
\begin{gathered}
L_{t^{\prime \prime}, \epsilon, \chi}^{1}=\{(x, y, z) \mid z=x \hat{+} y, x, y, z \in N\} . \\
L_{t, \gamma, \phi}^{1}=\{(x, y, z) \mid z=x \hat{+} y, x, y, z \in N\} . \\
L_{t^{\prime}, t^{\prime \prime}, \alpha}^{1}=\{(x, y, z) \mid z=(x \hat{+} y) / 2, x, y, z \in N\} . \\
L_{t^{\prime}, t, l}^{1}=\{(x, y, z) \mid z=(x \hat{+} y) / 2, x, y, z \in N\} .
\end{gathered}
$$

For any remaining triple of $T_{1}$ that looks like $\left\{t^{\prime}, X, Z\right\}$ such that there is some $Y \in V$ with $\left\{t^{\prime \prime}, X, Y\right\},\{t, Y, Z\} \in T_{1}$, define

$$
L_{t, X, Z}^{1}=\{(x, y, z) \mid z=x \hat{+} y,, x, y, z \in N\} .
$$

For any remaining triple of $T_{1}$ that looks like $\left\{t^{\prime \prime}, X, Z\right\}$ such that there is some $Y \in V$ with $\{t, X, Y\},\left\{\infty_{2}, Y, Z\right\} \in T_{1}$, define

$$
L_{t, X, Z}^{1}=\{(x, y, z) \mid z=x \hat{+} y \hat{+} 1, x, y, z \in N\} .
$$

Define

$$
\begin{aligned}
L_{\chi, t^{\prime}, t^{\prime \prime}}^{2} & =\{(x, y, z) \mid x=(y \hat{+} z) / 2+1, x, y, z \in N\} . \\
L_{\phi, t^{\prime \prime}, t}^{2} & =\{(x, y, z) \mid x=(y \hat{+} z) / 2+1, x, y, z \in N\} . \\
L_{\alpha, \eta, t^{\prime}}^{2} & =\{(x, y, z) \mid x=y \hat{+} z+1, x, y, z \in N\} . \\
L_{\iota, \kappa, t}^{2} & =\{(x, y, z) \mid z=x \hat{+} y+1, x, y, z \in N\} . \\
L_{\iota, t^{\prime \prime}, \lambda} & =\{(x, y, z) \mid z=(x \hat{+} y) / 2, x, y, z \in N\} .
\end{aligned}
$$

and for any remaining triple of $T_{2}$ that looks like $\left\{t^{\prime}, X, Z\right\}$ define

$$
L_{t^{\prime}, X, Z}^{2}=\{(x, y, z) \mid x=(y \hat{+} z) / 2+1, x, y, z \in N\} .
$$

For any remaining triple of $T_{2}$ that looks like $\left\{t^{\prime \prime}, X, Z\right\}$ define

$$
L_{t^{\prime \prime}, X, Z}^{2}=\{(x, y, z) \mid x=(y \hat{+} z) / 2, x, y, z \in N\} .
$$




\begin{tabular}{|c||c|c|c|}
\hline intersection number & $T_{0}$ & $T_{1}$ & $T_{2}$ \\
\hline \hline 3 & $\left\{t, t^{\prime}, t^{\prime \prime}\right\}$ & $\left\{\infty_{1}, t^{\prime \prime}, t\right\}$ & $\left\{\infty_{2}, t, t^{\prime}\right\}$ \\
\hline 3 & $\left\{t, \infty_{1}, \infty_{2}\right\}$ & $\left\{\infty_{1}, t^{\prime}, \infty_{2}\right\}$ & $\left\{\infty_{2}, \infty_{1}, t^{\prime \prime}\right\}$ \\
\hline 2 & $\left\{t^{\prime}, \infty_{1}, \alpha\right\}$ & $\left\{t^{\prime \prime}, t^{\prime}, \alpha,\right\}$ & $\left\{t, \infty_{1}, \alpha\right\}$ \\
\hline 2 & $\left\{t^{\prime}, \infty_{2}, \phi\right\}$ & $\left\{t^{\prime \prime}, \infty_{2}, \phi,\right\}$ & $\left\{t, t^{\prime \prime}, \phi\right\}$ \\
\hline 2 & $\left\{t^{\prime \prime}, \infty_{1}, \iota\right\}$ & $\left\{t, t^{\prime}, \iota,\right\}$ & $\left\{t^{\prime}, \infty_{1}, \iota\right\}$ \\
\hline 2 & $\left\{t^{\prime \prime}, \infty_{2}, \chi\right\}$ & $\left\{t, \infty_{2}, \chi,\right\}$ & $\left\{t^{\prime}, t^{\prime \prime}, \chi\right\}$ \\
\hline
\end{tabular}

Table 2: Triples of $T_{0}, T_{1}, T_{2}$ that Meet $P$ in 2 or 3 Points

For each $i \in \mathbb{Z} / 3 \mathbb{Z}$, construct a deleted symmetric square on $\mathcal{V}$ following the construction from Lemma 3.5 as we did in the earlier case of $m=7$. Call the resulting squares $B_{i}$ for $i \in \mathbb{Z} / 3 \mathbb{Z}$. We must show that $\left(\mathcal{V}, B_{0}, B_{1}, B_{2}\right)$ is a meager system. For easier reference, define the set $P=\left\{t, t^{\prime}, t^{\prime \prime}, \infty_{1}, \infty_{2}\right\}$. In this construction, define the notion of being derived as follows: Triples of $B_{0} \cup B_{1} \cup B_{2}$ can be categorized into three different types:

1. $S_{0}, S_{1}, S_{2}$.

2. triples whose form is $\{X, Y, Z\}$ with $\{X, Y, Z\} \in T_{i}$ for some $i$.

3. triples whose form is $\{X, X, Y\}$, for $\left\{X, Y, \infty_{i}\right\} \in T_{j}$ for some $i, j$.

A triple of the first type from $S_{0}, S_{1}$ or $S_{2}$ is said to be derived from $\left\{t, \infty_{1}, \infty_{2}\right\}$, $\left\{t^{\prime}, \infty_{1}, \infty_{2}\right\}$, or $\left\{t^{\prime \prime}, \infty_{1}, \infty_{2}\right\}$ of $T_{0}, T_{1}$, or $T_{2}$, respectively. A triple of the second type, $(x, y, z)$ in $B_{i}$ is said to be derived from $\{\operatorname{Pr}(x), \operatorname{Pr}(y), \operatorname{Pr}(z)\}$ of $T_{i}$. A triple of the third type from $B_{i}$, one of the form $\{X, X, Y\}$ where $\left\{X, Y, \infty_{j}\right\} \in T_{i}$ is said to be derived from $\left\{X, Y, \infty_{j}\right\}$ of $T_{i}$.

Claim: The systems $B_{0}, B_{1}$ and $B_{2}$ are pairwise really disjoint.

Proof of Claim. If there were a 3 -set $\{x, y, z\}$ such that $B_{i}$ and $B_{j}$ have triples composed of those elements for some $i \neq j$, then the triples must have the same type.

case 1: The triples are of type 1. However, this is absurd since the points of any two of $S_{0}, S_{1}$ and $S_{2}$ only have $\left\{\infty_{1}, \infty_{2}\right\}$ in common.

case 2: The triples are of type 2. Then the triples would have been derived from a common triple say $\{X, Y, Z\} \in T_{i} \cap T_{j}$. If $\{X, Y, Z\} \cap P=\emptyset$, then the subscripts assigned to form the triple in $B_{i}$ and $B_{j}$ come from disjoint squares, $L_{0}$ and $L_{1}$, respectively. Thus, it must be that $\{X, Y, Z\} \cap P \neq \emptyset$. By table 2, it follows that $\{X, Y, Z\}$ cannot intersect $P$ in more than one place ${ }^{4}$. So, suppose $Z \in P$. Then $\sigma_{1}$ and $\sigma_{2}$ move $Z$ to different places but keep $X$ and $Y$ fixed. It follows that $\{X, Y, Z\}$ cannot be a common triple between any two of $T_{0}, T_{1}$ and $T_{2}$ thus yielding a contradiction.

\footnotetext{
${ }^{4}$ The elements $\alpha, \chi, \iota, \phi$ are all distinct since there are no Paschs in $T_{0}$. Thus, the triple entries of table 2 are all distinct.
} 
case 3: The triple is of type 3. Let us assume that the $\operatorname{Pr}(x)=\operatorname{Pr}(y)$ and set $X=\operatorname{Pr}(x)$ and $Z=\operatorname{Pr}(z)$. Even if we identify $\infty_{1}$ and $\infty_{2}$ as the same point, the triples of table 2 remain distinct. It follows that $X, Y \notin P$.

If $\{i, j\}=\{0,1\}$ then it must be that $\left\{X, Y, \infty_{2}\right\} \in T_{0} \cap T_{1}$. The disjoint Valek squares, $R^{0}$ and $R^{1}$, are used to assign the subscripts for the corresponding triples of $B_{0}$ and $B_{1}$, respectively (except when $\{X, Y\}=\{\iota, \lambda\}$, but in this case the Valek squares $R^{0}$ and $R^{1}$ switch roles). Then the triples would not have come from the same 3 -set which gives us a contradiction.

Similarly, if $\{i, j\}=\{0,2\}$ then it must be that $\left\{X, Y, \infty_{1}\right\} \in T_{0} \cap T_{2}$. The disjoint Valek squares, $R^{0}$ and $R^{2}$, are used to assign the subscripts for the corresponding triples of $B_{0}$ and $B_{2}$, respectively. It follows that the triples would not have come from the same 3 -set, a contradiction.

Lastly, if $\{i, j\}=\{1,2\}$ then it must be that $\left\{X, Y, \infty_{i}\right\} \in T_{0}$ and $\left\{X, Y, \infty_{j}\right\} \in T_{1}$ for $i \neq j$. Since disjoint Valek squares are used to assign the subscripts for the corresponding triples of $B_{1}$ and $B_{2}$, it follows that the triples would not have come from the same 3 -set, a contradiction.

So it is clear that the systems $B_{0}, B_{1}$ and $B_{2}$ are really disjoint. Also, the only nonsupersymmetric triples for the systems lie in their subsystems $S_{0}, S_{1}$ and $S_{2}$, respectively. Also, any ordered triple of $V_{i}$ does not lie in $B_{j}$ if $i \neq j$. Thus the system $\left(\mathcal{V}, B_{0}, B_{1}, B_{2}\right)$ satisfies the hypothesis of Lemma 8.1. Thus we only have to show that the system contains no $M_{3}^{\prime}$ or $Q$ configurations. Suppose on the contrary, that there is an $M_{3}^{\prime}$ configuration:

$$
(x, y, z),(y, w, x) \in S_{i},(x, z, v) \in B_{i+1} \text {, and }(x, v, w) \in B_{i+2} .
$$

Then we have the following cases to consider:

case 1: $i=0$. Then $x, y, z, w \in\left\{t, \infty_{1}, \infty_{2}\right\}$. It follows that $\operatorname{Pr}(v) \in\left\{t^{\prime}, t^{\prime \prime}\right\}$. If $v=t^{\prime}$, then after investigating the forms and filling the subscripts, we arrive at:

$$
\left(\infty_{2}, t_{-3}, \infty_{1}\right),\left(t_{-3}, t_{1}, \infty_{2}\right) \in S_{0},\left(\infty_{2}, \infty_{1}, t_{-3 / 2}^{\prime}\right\} \in B_{1},\left(\infty_{2}, t_{-3 / 2}^{\prime}, t_{1}\right) \in B_{2} .
$$

The idempotent Valek square $R^{0}$ defines how the subscripts are assigned on the triple $\left\{\infty_{2}, t^{\prime}, t\right\} \in B_{2}$. This implies that $-3 / 2=1$ which is impossible since $n+2 \neq 5$.

Similarly, if $v=t^{\prime \prime}$ then we arrive at the configuration:

$$
\left(\infty_{1}, t_{-3}, t_{-5 / 2}\right),\left(t_{-3}, \infty_{2}, \infty_{1}\right) \in S_{0},\left(\infty_{1}, t_{-5 / 2}, t_{0}^{\prime \prime}\right\} \in B_{1},\left(\infty_{1}, t_{0}^{\prime \prime}, \infty_{2}\right) \in B_{2} .
$$

The idempotent Valek square $R^{0}$ defines how the subscripts are assigned on the triple $\left\{\infty_{1}, t, t^{\prime \prime}\right\} \in B_{1}$. This implies that $0=-5 / 2$ which is impossible since $n+2 \neq 5$. 
case 2: $i=1$. Then $\operatorname{Pr}(v)=t$. It follows that the configuration looks like:

$$
\left(\infty_{2}, t_{0}^{\prime}, t_{-1 / 2}^{\prime}\right),\left(t_{0}^{\prime}, \infty_{1}, \infty_{2}\right) \in S_{1},\left(\infty_{2}, t_{-1 / 2}^{\prime}, t_{-3}\right\} \in B_{2},\left(\infty_{2}, t_{-3}, \infty_{1}\right) \in B_{0} .
$$

The triple $\left\{\infty_{2}, t^{\prime}, t\right\} \in T_{2}$ has subscripts assigned from the idempotent Valek square $R^{0}$. Thus $-1 / 2=-3$ which is impossible since $n+2 \neq 5$.

case 3: $i=2$. Then $\operatorname{Pr}(v)=t$. It follows that the configuration looks like:

$$
\left(\infty_{1}, t_{0}^{\prime \prime}, \infty_{2}\right),\left(t_{0}^{\prime \prime}, t_{-4}^{\prime \prime}, \infty_{1}\right) \in B_{2},,\left(\infty_{1}, \infty_{2}, t_{-3 / 2}\right) \in B_{0},\left(\infty_{1}, t_{-3 / 2}, t_{-4}^{\prime \prime}\right) \in B_{1} .
$$

Then we require $-3 / 2=-4$ which cannot be satisfied since $n+2 \neq 5$.

Now we must check that there are no $Q$ configurations. Assume, on the contrary, that there is a $Q$ configuration in $\left(\mathcal{V}, B_{0}, B_{1}, B_{2}\right)$. We consider the following cases:

1. The triple from $B_{0}$ is of type 1 . Then the form of the triple consists of elements from the set $\left\{t, \infty_{1}, \infty_{2}\right\}$. This leads to only two possibilities: The form of the $Q$ configurations must look like one of the following:

$$
\begin{gathered}
\left(\infty_{1}, \infty_{2}, t\right) \in \operatorname{Pr}\left(B_{0}\right),\left(\infty_{1}, t, t^{\prime \prime}\right) \in \operatorname{Pr}\left(B_{1}\right),\left(\infty_{1}, t^{\prime \prime}, \infty_{2}\right) \in \operatorname{Pr}\left(B_{2}\right), \text { or } \\
\left(\infty_{2}, t, \infty_{1}\right) \in \operatorname{Pr}\left(B_{0}\right), \quad\left(\infty_{2}, \infty_{1}, t^{\prime}\right) \in \operatorname{Pr}\left(B_{1}\right),\left(\infty_{2}, t^{\prime}, t\right) \in \operatorname{Pr}\left(B_{2}\right) .
\end{gathered}
$$

Filling in the subscripts in the first case gives us:

$$
\left(\infty_{1}, \infty_{2}, t_{-3 / 2}\right) \in B_{0},\left(\infty_{1}, t_{-3 / 2}, t_{0}^{\prime \prime}\right) \in B_{1},\left(\infty_{1}, t_{0}^{\prime \prime}, \infty_{2}\right) \in B_{2}
$$

The Valek square $R^{0}$ that determined the subscripts of the triple form $\left(\infty_{1}, t, t^{\prime \prime}\right)$ is idempotent and thus would have the subscripts of $t$ and $t^{\prime \prime}$ equal. Then $-3 / 2=0$ which is impossible since $n+2 \neq 3$.

Filling in the subscripts in the second case gives us:

$$
\left(\infty_{2}, t_{-3}, \infty_{1}\right) \in B_{0},\left(\infty_{2}, \infty_{1}, t_{-3 / 2}^{\prime}\right) \in B_{1},\left(\infty_{2}, t_{-3 / 2}^{\prime}, t_{-3}\right) \in B_{2}
$$

As in the first case, the Valek square $R^{0}$ that determined the subscripts of the triple form $\left(\infty_{2}, t^{\prime}, t\right)$ is idempotent and thus would have the subscripts of $t^{\prime}$ and $t$ equal. Then $-3 / 2=3$ which is impossible since $n+2 \neq 3$.

2. The triple from $B_{0}$ is of type 2. Consider the following cases:

(a) The triple from $B_{0}$ meets $P$ in three points. Then the only possible form for a $Q$ configuration is:

$$
\left(t, t^{\prime}, t^{\prime \prime}\right) \in \operatorname{Pr}\left(B_{0}\right),\left(t, t^{\prime \prime}, t\right) \in \operatorname{Pr}\left(B_{1}\right),\left(t, t, t^{\prime}\right) \in \operatorname{Pr}\left(B_{2}\right) .
$$

(Notice that the above configuration will not exist if $\left(t, t^{\prime \prime}\right) \notin \Omega_{1}$ or if $\left(t, t^{\prime}\right) \notin$ $\Omega_{2}$.) Filling in the subscripts gives us:

$$
\left(t_{x}, t_{y}^{\prime}, t_{z}^{\prime \prime}\right) \in B_{0},\left(t_{x}, t_{z}^{\prime \prime}, t_{w}\right) \in B_{1},\left(t_{x}, t_{w}, t_{y}^{\prime}\right) \in B_{2}
$$


Since the subscripts assigned to the form $\left(t, t^{\prime \prime}, t\right) \in \operatorname{Pr}\left(B_{1}\right)$ and the subscripts assigned to the form $\left(t, t, t^{\prime}\right) \in \operatorname{Pr}\left(B_{2}\right)$ come from the same Valek square $R^{0}$, it follows that $y=z$. Since $x=(y \hat{+} z) / 2$, it follows that $x=y=z$ which cannot happen since $R^{0}$ is idempotent.

(b) The triple from $B_{0}$ meets $P$ in exactly two points. Since $T_{0}$ has no Paschs, it is clear that it cannot contain any of the triples $\{t, \alpha, \iota\}$ or $\{t, \phi, \chi\}$. With this in mind, it is easy to see that there are no possible forms for $Q$ configurations in this case.

(c) The triple from $B_{0}$ meets $P$ in exactly one point. It is easy to see the elements of the form of the triple does not contain $\infty_{1}$ or $\infty_{2}$. Thus we only have to consider the following three cases:

i. The triple from $B_{0}$ has the form with elements from the set $\{t, X, Y\}$ with $X, Y \notin P$. If the $Q$ configuration has the form $(t, X, Y) \in \operatorname{Pr}\left(B_{0}\right)$, $(t, Y, W) \in \operatorname{Pr}\left(B_{1}\right)$ and $(t, W, X) \in \operatorname{Pr}\left(B_{2}\right)$, then consider the following. If $W \in P$, it follows that $\{t, \alpha, \chi\} \in T_{0}$, but this is impossible based on the fact that we chose points $\left\{t, t^{\prime}, t^{\prime \prime}, \infty_{1}, \infty_{2}, \alpha, \beta\right\}$ in such a way so that they do not form a mitre in $T_{0}$. Thus it must be that $W \notin P$. Then there is a Pasch in $T_{0}$ :

$$
\left\{t^{\prime \prime}, Y, W\right\},\left\{t^{\prime \prime}, t^{\prime}, t\right\},\{X, Y, t\},\left\{t^{\prime}, X, W\right\} .
$$

Keeping in mind that $(\chi, \delta) \notin \Omega_{2}$ and $(\alpha, \beta) \notin \Omega_{1}$ it is clear that the only possible forms for the $Q$ configuration are:

$$
\begin{array}{r}
(\chi, \delta, t) \in \operatorname{Pr}\left(B_{0}\right),\left(\chi, t, \infty_{2}\right) \in \operatorname{Pr}\left(B_{1}\right),\left(\chi, \infty_{2}, \delta\right) \in \operatorname{Pr}\left(B_{2}\right) \text {, or } \\
(\alpha, t, \beta) \in \operatorname{Pr}\left(B_{0}\right),\left(\alpha, \beta, \infty_{1}\right) \in \operatorname{Pr}\left(B_{1}\right),\left(\alpha, \infty_{1}, t\right) \in \operatorname{Pr}\left(B_{2}\right) .
\end{array}
$$

Filling in the subscripts in the first case, we arrive at the $Q$ configuration:

$$
\left(\chi_{x}, \delta_{x}, t_{x}\right) \in B_{0},\left(\chi_{x}, t_{x}, \infty_{2}\right) \in B_{1},\left(\chi_{x}, \infty_{2}, \delta_{x}\right) \in B_{2}
$$

for some $x \in N$. However, this cannot happen since the Latin square that defined the subscripts assigned to the triples of the form $(\chi, \delta, t) \in T_{0}$ avoid $(x, x, x)$. A similar argument holds for the second case above.

ii. The triple from $B_{0}$ has the form with elements from the set $\left\{t^{\prime}, X, Y\right\}$ with $X, Y \notin P$. If the triple has the form $\left(X, Y, t^{\prime}\right)$, then it must be that $X=\iota$ and $Y=\kappa$. This gives us the $Q$ configuration:

$$
\left(\iota_{x}, \kappa_{y}, t_{z}^{\prime}\right) \in B_{0},\left(\iota_{x}, t_{z}^{\prime}, t_{w}\right) \in B_{1},\left(\iota_{x}, t_{w}, \kappa_{y}\right) \in B_{2}
$$

where $x, y, z, w \in N$ with:

$$
\begin{aligned}
z & =x \hat{+} y \\
x & =(z \hat{+} w) / 2 \\
x & =w \hat{+} y \hat{+} 1
\end{aligned}
$$


which has no solution.

If the triple has the form $\left(X, t^{\prime}, Y\right)$, then it must be that $X=\chi$ and $Y=\epsilon$. This gives us the $Q$ configuration:

$$
\left(\chi_{x}, t_{y}^{\prime}, \epsilon_{z}\right) \in B_{0},\left(\chi_{x}, \epsilon_{z}, t_{w}^{\prime \prime}\right) \in B_{1},\left(\chi_{x}, t_{w}^{\prime \prime}, t_{y}^{\prime}\right) \in B_{2}
$$

where $x, y, z, w \in N$ with:

$$
\begin{aligned}
y & =x \hat{+} z \\
x & =z \hat{+} w \\
x & =(w \hat{+} y) / 2 \hat{+} 1
\end{aligned}
$$

which also cannot be satisfied.

If the triple has the form $\left(t^{\prime}, X, Y\right)$, then the form of the $Q$ configuration must be:

$$
\left(t^{\prime}, X, Y\right) \in \operatorname{Pr}\left(B_{0}\right), \quad\left(t^{\prime}, Y, Z\right) \in \operatorname{Pr}\left(B_{1}\right),\left(t^{\prime}, Z, X\right) \in \operatorname{Pr}\left(B_{2}\right)
$$

where $Z$ is some element of $V, X$ and $Y$ are distinct and $\left\{\infty_{1}, Y, Z\right\}$, $\left\{t^{\prime \prime}, Z, X\right\} \in T_{0}$. Notice that this implies that $\left\{t^{\prime}, \alpha, \eta\right\} \neq\left\{t^{\prime}, Z, X\right\}$. Thus we have the following $Q$ configuration:

$$
\left(t_{x}^{\prime}, X_{y}, Y_{z}\right) \in B_{0},\left(t_{x}^{\prime}, Y_{z}, Z_{w}\right) \in B_{1},\left(t_{x}^{\prime}, Z_{w}, X_{y}\right) \in B_{2}
$$

where $x, y, z, w \in N$ with:

$$
\begin{aligned}
x & =y \hat{+} z \\
w & =x \hat{+} z \\
x & =(w \hat{+} y) / 2 \hat{+} 1
\end{aligned}
$$

which cannot happen.

iii. The triple from $B_{0}$ has the form with elements from the set $\left\{t^{\prime \prime}, X, Y\right\}$ with $X, Y \notin P$. If the triple has the form $\left(X, Y, t^{\prime \prime}\right)$, then it must be that $X=\alpha$ and $Y=\eta$. This gives us the $Q$ configuration:

$$
\left(\alpha_{x}, \eta_{y}, t_{z}^{\prime \prime}\right) \in B_{0},\left(\alpha_{x}, t_{z}^{\prime \prime}, t_{w}^{\prime}\right) \in B_{1},\left(\alpha_{x}, t_{w}^{\prime}, \eta_{y}\right) \in B_{2}
$$

where $x, y, z, w \in N$ with:

$$
\begin{aligned}
z & =x \hat{+} y \\
x & =(z \hat{+} w) / 2 \\
x & =w \hat{+} y \hat{+} 1
\end{aligned}
$$

which has no solution. 
If the triple has the form $\left(X, t^{\prime \prime}, Y\right)$, then it must be that $X=\phi$ and $Y=\gamma$. This gives us the $Q$ configuration:

$$
\left(\phi_{x}, t_{y}^{\prime \prime}, \gamma_{z}\right) \in B_{0},\left(\phi_{x}, \gamma_{z}, t_{w}\right) \in B_{1},\left(\phi_{x}, t_{w}, t_{y}^{\prime \prime}\right) \in B_{2}
$$

where $x, y, z, w \in N$ with:

$$
\begin{aligned}
y & =x \hat{+} z \\
x & =z \hat{+} w \\
x & =(w \hat{+} y) / 2 \hat{+} 1
\end{aligned}
$$

which also cannot be satisfied.

If the triple has the form $\left(t^{\prime \prime}, X, Y\right)$, then the form of the $Q$ configuration must be:

$$
\left(t^{\prime \prime}, X, Y\right) \in \operatorname{Pr}\left(B_{0}\right),\left(t^{\prime \prime}, Y, Z\right) \in \operatorname{Pr}\left(B_{1}\right),\left(t^{\prime \prime}, Z, X\right) \in \operatorname{Pr}\left(B_{2}\right)
$$

where $Z$ is some element of $V, X$ and $Y$ are distinct and $\left\{t^{\prime}, Y, Z\right\}$, $s\left\{\infty_{2}, Z, X\right\} \in T_{0}$. Notice that this implies that $\left\{t^{\prime \prime}, \chi, \epsilon\right\} \neq\left\{t^{\prime \prime}, Z, X\right\}$. Thus we have the following $Q$ configuration:

$$
\left(t_{x}^{\prime \prime}, X_{y}, Y_{z}\right) \in B_{0},\left(t_{x}^{\prime \prime}, Y_{z}, Z_{w}\right) \in B_{1},\left(t_{x}^{\prime \prime}, Z_{w}, X_{y}\right) \in B_{2}
$$

where $x, y, z, w \in N$ with:

$$
\begin{aligned}
x & =y \hat{+} z \\
w & =x \hat{+} z \hat{+} 1 \\
x & =(w \hat{+} y) / 2
\end{aligned}
$$

which cannot happen.

(d) The triple from $B_{0}$ is disjoint from $P$. Let the form of the triple be composed of the elements $X, Y, Z$. Then these three elements are distinct, and, by the fact that $\sigma_{1}$ and $\sigma_{2}$ fix $V \backslash P$, the other two triples from $B_{1}$ and $B_{2}$ in the $Q$ configuration must also have forms from the set $\{X, Y, Z\}$ which is impossible for the configuration.

3. The triple from $B_{0}$ is of type 3. Then the form of the triple is composed of elements $X, Y$ for some $X, Y \in V$. If $X, Y \cap P=\emptyset$, then we have the following two cases:

(a) The triple was derived from a triple $\left\{X, Y, \infty_{1}\right\} \in T_{0}$. Then there are triples $\left\{X, Y, t^{\prime}\right\} \in T_{1}$ and $\left\{X, Y, \infty_{1}\right\} \in T_{2}$. The only possible forms of triples in the $Q$ configuration are:

$$
\begin{gathered}
(X, X, Y) \in \operatorname{Pr}\left(B_{0}\right),\left(X, Y, t^{\prime}\right) \in \operatorname{Pr}\left(B_{1}\right),\left(X, t^{\prime}, X\right) \in \operatorname{Pr}\left(B_{2}\right), \text { or } \\
(X, Y, X) \in \operatorname{Pr}\left(B_{0}\right),\left(X, X, \infty_{1}\right) \in \operatorname{Pr}\left(B_{1}\right),\left(X, \infty_{1}, Y\right) \in \operatorname{Pr}\left(B_{2}\right) .
\end{gathered}
$$


In the first case, it is clear that $T_{2}$ has a triple $\left\{X, t^{\prime}, \infty_{1}\right\}$ or $\left\{X, t^{\prime}, \infty_{2}\right\}$ which in turn implies that $X=\iota$ or $X=t$. Since we are assuming in this case that $X \notin P$, it must be that $X=\iota$. Then $Y=t$ which cannot happen. In the second case, it must be that $B_{1}$ has a triple of the form $\left\{X, X, \infty_{1}\right\}$ which can only be satisfied if $X=t^{\prime}$ which cannot happen by assumption.

(b) The triple was derived from a triple $\left\{X, Y, \infty_{2}\right\} \in T_{0}$. The only possible forms of triples in the $Q$ configuration are:

$$
\begin{array}{r}
(X, X, Y) \in \operatorname{Pr}\left(B_{0}\right),\left(X, Y, \infty_{2}\right) \in \operatorname{Pr}\left(B_{1}\right),\left(X, \infty_{2}, X\right) \in \operatorname{Pr}\left(B_{2}\right), \\
(X, Y, X) \in \operatorname{Pr}\left(B_{0}\right),\left(X, X, t^{\prime \prime}\right) \in \operatorname{Pr}\left(B_{1}\right),\left(X, t^{\prime \prime}, Y\right) \in \operatorname{Pr}\left(B_{2}\right) .
\end{array}
$$

In the first case, it is clear that $\operatorname{Pr}\left(B_{2}\right)$ has a triple $\left(X, X, \infty_{2}\right)$ which can only be satisfied if $X=t^{\prime \prime}$ which cannot happen by assumption. In the second case, it must be that $B_{1}$ has a triple of the form $\left(X, X, t^{\prime \prime}\right)$ which must have been derived from the triple $\left(X, t^{\prime \prime}, \infty_{1}\right)$ or $\left(X, t^{\prime \prime}, \infty_{2}\right)$. In either case, $X=t$ or $X=\phi$. However, $X=t$ cannot happen by assumption, and if $X=\phi$, then $Y=t^{\prime}$ which also cannot happen by assumption.

If $\{X, Y\} \cap P \neq \emptyset$, then it must be that the intersection consists of only one point (cf. Table 2) and furthermore, that point must be in $\left\{t, t^{\prime}, t^{\prime \prime}\right\}$. Keeping in mind that $\alpha, \chi, \phi$ and $\iota$ are distinct elements and that $\left(\phi, t^{\prime}\right) \notin \Omega_{0}$, it is easy to check that there is only one possible feasible form for the $Q$ configuration:

$$
\left(\iota, t^{\prime \prime}, \iota\right) \in \operatorname{Pr}\left(B_{0}\right),(\iota, \iota, \lambda) \in \operatorname{Pr}\left(B_{1}\right),\left(\iota, \lambda, t^{\prime \prime}\right) \in \operatorname{Pr}\left(B_{2}\right) .
$$

(Notice that these forms do not exist if $(\iota, \lambda) \notin \Omega_{1}$ or $\left(\iota, t^{\prime \prime}\right) \notin \Omega_{0}$.) Filling in the subscripts gives us:

$$
\left(\iota_{x}, t_{y}^{\prime \prime}, \iota_{z}\right) \in B_{0},\left(\iota_{x}, \iota_{z}, \lambda_{y}\right) \in B_{1},\left(\iota_{x}, \lambda_{y}, t_{y}^{\prime \prime}\right) \in B_{2} .
$$

It follows that $x=y$ and thus $x=y=z$ which cannot happen.

Thus there are no $Q$ configurations in the system $\left(\mathcal{V}, B_{0}, B_{1}, B_{2}\right)$ and hence it is a meager system of order $m n+2$.

\section{References}

[1] E. F. Assmus, Linearly Derived Steiner Triple Systems, Designs, Codes and Cryptography, 13(1998), 31-49.

[2] C. Colbourn, E. Mendelsohn, A. Rosa, and J. Širán̆ , Anti-Mitre Steiner Triple Systems, Graphs and Combinatorics, 10(1994), 215-224.

[3] J. Dénes and A. D. Keedwell, "Latin Squares: New Developments in the Theory and Applications", Annals of Discrete Mathematics, 46, Elsevier Science Publishing Company, INC., (1991). 
[4] Paul Erdős, Problems and Results in Combinatorial Analysis, Creation in Mathematics, 9(1976), 25.

[5] Yuichiro Fujiwara, Infinite Classes of Anti-Mitre and 5-sparse Steiner Triple Systems, to be published in: Journal of Combinatorial Designs(2005).

[6] M.J. Grannell, T.S. Griggs, and C.A. Whitehead, The Resolution of the Anti-Pasch Conjecture, Journal of Combinatorial Designs, 8(2000), 300-309.

[7] M.J. Grannell, T.S. Griggs, and J.S. Phelan, A New Look At An Old Construction For Steiner Triple Systems, Ars Combinatoria, 25A(1988), 55-60.

[8] T.S. Griggs, J. Murphy, and S. Phelan, Anti-Pasch Steiner Triple Systems, Journal of Combinatorics Information \& System Sciences, 15(1990), 79-84.

[9] Kenneth Ireland and Michael Rosen, "A Classical Introduction to Modern Number Theory", Springer-Verlag, New York, (1998).

[10] A.C.H. Ling, A Direct Product Construction For 5-Sparse Triple Systems, Journal of Combinatorial Designs, 5(1997), 443-447.

[11] A.C.H. Ling, C.J. Colbourn, M.J. Grannell, and T.S. Griggs, Construction Techniques for Anti-Pasch Steiner Triple Systems, Journal of the London Mathematical Society, 61(2000), 641-657.

[12] Jia-Xi Lu, On Large Sets of Disjoint Steiner Triple Systems, Journal of Combinatorial Theory, Series A, 37(1984), 136-163.

[13] R.M. Wilson, Nonisomorphic Steiner Triple Systems, Math. Z., 135(1974), 303-313. 